\title{
A multiple marker level-set method for simulation of deformable fluid particles
}

\author{
Néstor Balcázar ${ }^{\mathrm{a}, *}$, Oriol Lehmkuhl ${ }^{\mathrm{a}, \mathrm{b}}$, Joaquim Rigola ${ }^{\mathrm{a}}$, Assensi Oliva ${ }^{\mathrm{a}, *}$ \\ ${ }^{a}$ Heat and Mass Transfer Technological Center (CTTC), Universitat Politècnica de \\ Catalunya - BarcelonaTech (UPC) ETSEIAT, Colom 11, 08222 Terrassa (Barcelona), \\ Spain.E-mail: cttc@upc.edu \\ ${ }^{b}$ Termo Fluids, S.L., Avda Jacquard 97 1-E, 08222 Terrassa (Barcelona), Spain. \\ E-mail: termofluids@termofluids.com
}

\begin{abstract}
A novel multiple marker level-set method is introduced for Direct Numerical Simulation of deformable fluid particles (bubbles and droplets), which is integrated in a finite-volume framework on collocated unstructured grids. Each fluid particle is described by a separate level-set function, thus, different interfaces can be solved in the same control volume, avoiding artificial and potentially unphysical coalescence of fluid particles. Therefore, bubbles or droplets are able to approach each other closely, within the size of one grid cell, and can even collide. The proposed algorithm is developed in the context of the conservative level-set method, whereas, surface tension is modeled by the continuous surface force approach. The pressure-velocity coupling is solved by the fractional-step projection method. For validation of the proposed numerical method, the gravity-driven impact of a droplet on a liquid-liquid interface is studied; then, the binary droplet collision with bouncing outcome is examined, and finally, it is applied on simulation of gravity-driven bubbly flow in a vertical column.
\end{abstract}

Keywords: level-set method; multiple marker; bubbles; droplets; two-phase flow

\footnotetext{
*Corresponding author. Fax: +(34) 937398920

Email addresses: nestor@cttc.upc.edu (Néstor Balcázar), cttc@cttc.upc.edu (Assensi Oliva )
} 


\section{Introduction}

Bubbles and droplets are ubiquitous and they play an important role in many natural and industrial processes. Among them one can mention steam generators in nuclear plants, rocket engines, boiling heat transfer, microfluidic applications, unit operations in chemical engineering such as distillation, absorption, liquid-liquid extraction, heterogeneous catalysis and bioreactors (Mudde, 2005). Thus, the practical implications of a better understanding and predictive capabilities of droplet and bubble flows is enormous. Furthermore, all these applications have stimulated basic research on bubble and droplet dynamics, however, although numerous experimental and numerical investigations have provided considerable insight into the mechanisms governing droplet and bubble flows, many challenging problems still remain as pointed out in recent publications (Mudde , 2005; Jamet et al. , 2010; Tryggvason et al., 2010, 2013).

The development of computers has promoted Direct Numerical Simulation (DNS) of the Navier-Stokes equations as another means of performing controlled experiments (Tryggvason et al., 2010, 2013), providing a good way to non-invasive measure of droplet and bubble flows, although computationally demanding. Thus, DNS allows us to control the size distribution of bubbles or droplets, their deformability, whether they coalesce or not, and modify the flow conditions (Tryggvason et al., 2013). For DNS of multiphase flow problems with two immiscible fluid phases, standard Eulerian descriptions are often adopted together with interface-capturing methods such as volume-of-fluid (Scardovelli et al., 1999) and level-set (Osher et al., 1988; Sussman et al., 1994) methods, or front-tracking methods (Unverdi et al., 1992; Tryggvason et al., 2001). A review of advantage and disadvantages of the aforementioned techniques, in the context of simulation of multiphase flows with sharp interfaces is given in Van Sint Annaland et al. (2005). The main advantage of using a front-tracking method is that bubbles or droplets do not coalesce unless a specific merge condition is implemented. Especially for bubble swarm simulations this is an important aspect. On the other hand, in conventional level-set and volume-of-fluid methods, two fluid particles will automatically coalesce when their interfaces are close enough from each other. Indeed, up to now, most research on systems with multiple droplets or bubbles have been performed using the front-tracking method (Roghair et al., 2011; Tryggvason et al., 2013).

In spite of the rapid progress in DNS of complex multiphase flows, captur- 
ing the dynamics of deformable fluid-fluid interfaces in close hydrodynamic interaction is extremely difficult challenge for any numerical method because of the critical role played by the very thin near contact lubrication zone between the interfaces, where large pressures develop and resist coalescence sometimes preventing it altogether. In this regard, the front tracking method introduced by Unverdi et al. (1992) and Tryggvason et al. (2001) has been used to perform simulations where the aforementioned issues have an important impact on the numerical results. For instance, Nobari et al. (1996) and Nobari and Tryggvason (1996) have employed the front tracking method to perform numerical simulations of binary drop collisions. Thomas et al. (2010) examine the collision of a fluid drop with a wall and develop a multiscale approach to compute the flow in the film between the drop and the wall. In addition, the front tracking method has been extensively used in simulation of homogeneous bubbly flows, for instance in Tryggvason et al. (2013) and Roghair et al. (2011). Pan et al. (2008) carried out numerical simulations and experiments of the dynamics of head-on collision between two identical droplets. Using this empirical information as an input, the simulated collision images obtained by using a front tracking method were found to agree well with the experimental observations.

Regarding interface capturing methods (e.g. level-set and volume-offluid), Coyajee and Boersma (2009) have extended the level-set/volume-offluid method of Sussman and Puckett (2000) to a multiple marker approach in order to avoid the numerical merging of interfaces. This method was extended by Kwakkel et al. (2013) to include a coalescence criterion based on a film drainage model to predict if and when two colliding droplets will coalesce. In the same line, Focke et al. (2013) adapted a multiple marker approach to a volume-of-fluid method in order to deploy an algorithm for the temporal suppression of the coalescence in binary droplet collisions, where one droplet is composed of a high viscous liquid and the other one is of lower viscosity. In addition some previous works have been reported on the use of multiple level sets to represent various regions, for instance Merriman et al. (1994); Ruuth (1997); Smith et al. (2002) have extended the method of Osher et al. (1988) in order to solve the motion of multiple junctions. Losasso et al. (2006) have proposed an approach to extend the particle level-set method to the simulation of multiple fluids with different viscosities, densities or viscoelastic properties. Kim (2010) address the problem of multi-fluid simulations using a regional level set method for simulations of many immiscible materials. The last works, Losasso et al. (2006) and Kim (2010), have been designed for 
graphics applications. Wang and Wang (2004) have addressed the problem of structural shape and topology optimization in a multi-material domain, using $m$ level-set functions to represent a structure of $n=2^{m}$ different material phases. In some special cases, such as the head-on collision between two equal sized droplets, the numerical coalescence which is inherent to interfacecapturing methods can be controlled by manipulating the boundary conditions at the symmetry plane in between the two droplets (Jiang and James, 2007; Li and Fritsching, 2011). Zheng et al. (2005) and Ceniceros et al. (2010) have explore adaptive mesh refinement algorithms in order to capture thin films formed when two masses collide. However, this films can become very thin and it get down to just about few nanometers before it ruptures, therefore this approach would lead to a huge computational effort due to a large number of grid cells or levels of adaptive grid refinement which is currently not practical in a Direct Numerical Simulation even with high performance computing techniques.

The method presented in this paper is designed to overcome the above mentioned issues in a computationally efficient and robust manner. The concept of multiple marker is coupled with a conservative level-set method introduced in Balcázar et al. (2014) to deploy a numerical algorithm capable to simulate the dynamics of multiple bubbles and droplets on complex spatial domains without numerical merging of the interfaces. Thus, using the conservative level-set method (Olsson et al., 2005), mass conservation problem that is known to affect standard level-set formulations (Osher et al., 1988; Sussman et al., 1994) is circumvented. Moreover, the unstructured formulation of the multiphase solver allow us an efficient distribution of the mesh on complex domains (Balcázar et al., 2014). The extended algorithm is based on the idea of describing separate interfaces with different level-set functions to prevent numerical and potentially unphysical coalescence of bubbles and droplets, without excessive mesh refinement. Therefore, fluid particles are able to approach each other closely, within the size of one grid cell, and can even collide. Another important aspect is that the volume of the deformable fluid particles remain constant throughout the simulation, this is an important aspect on numerical simulation of homogeneous bubble swarms. The surface tension force and the interfacial geometrical properties are computed from the tracked interface, by using the continuum surface force model (CSF) introduced by (Brackbill et al., 1992), furthermore, the aforementioned approach has been adapted to be applicable in the context of the multiple marker level-set method. 
This paper is organized in the following order: The governing equations employed in this study are given in section 2. Section 3 is devoted to a description of the numerical method, while the simulation results are presented in section 4. Finally, concluding remarks are presented in section 5.

\section{Governing equations}

\subsection{Incompressible two-phase flow}

The Navier-Stokes equations for the dispersed fluid in $\Omega_{d}$ and continuous fluid in $\Omega_{c}$ can be combined into a set of equations in an entire domain $\Omega=\Omega_{d} \cup \Omega_{c}$, with a singular source term for the surface tension force at the interface $\Gamma$ :

$$
\frac{\partial}{\partial t}(\rho \mathbf{v})+\nabla \cdot(\rho \mathbf{v v})=-\nabla p+\nabla \cdot \mu\left(\nabla \mathbf{v}+(\nabla \mathbf{v})^{T}\right)+\rho \mathbf{g}+\mathbf{f}_{\sigma}
$$

where $\mathbf{v}$ and $p$ denote the fluid velocity and pressure field respectively, $\rho$ is the fluid density, $\mu$ is the dynamic viscosity, $\mathbf{g}$ is the gravitational acceleration, and $\mathbf{f}_{\sigma}$ is the surface tension force. Because of incompressibility, the velocity field is divergence-free:

$$
\nabla \cdot \mathbf{v}=0
$$

Moreover, physical properties change discontinuously across the interface:

$$
\begin{array}{r}
\rho=\rho_{d} H_{d}+\rho_{c}\left(1-H_{d}\right) \\
\mu=\mu_{d} H_{d}+\mu_{c}\left(1-H_{d}\right)
\end{array}
$$

with $\rho_{d}, \rho_{c}$ and $\mu_{d}, \mu_{c}$ the densities and viscosities of the disperse and continuous fluids, respectively. $H_{d}$ the Heaviside step function that is one in $\Omega_{d}$ and zero elsewhere.

\subsection{Multiple marker level-set method}

In level-set methods (Osher et al., 1988; Sussman et al., 1994), merging of interfaces happens automatically whenever two interfaces come within one grid cell of each other. To circumvent this issue, multiple markers, $\phi_{i}$, are introduced to represent each subdomain, $\Omega_{i}$, in the dispersed phase $\Omega_{d}=$ $\left\{\Omega_{1}, \ldots, \Omega_{n_{d}}\right\}$, where $n_{d}$ is the number of separate regions included in $\Omega_{d}$ (see Fig. 1). Thus, the inclusion of separate markers will permit to solve two or more interfaces, $\Gamma_{i}$, at the same grid cell. In the present work, a multiple tracking methodology is introduced in the context of the conservative level-set 


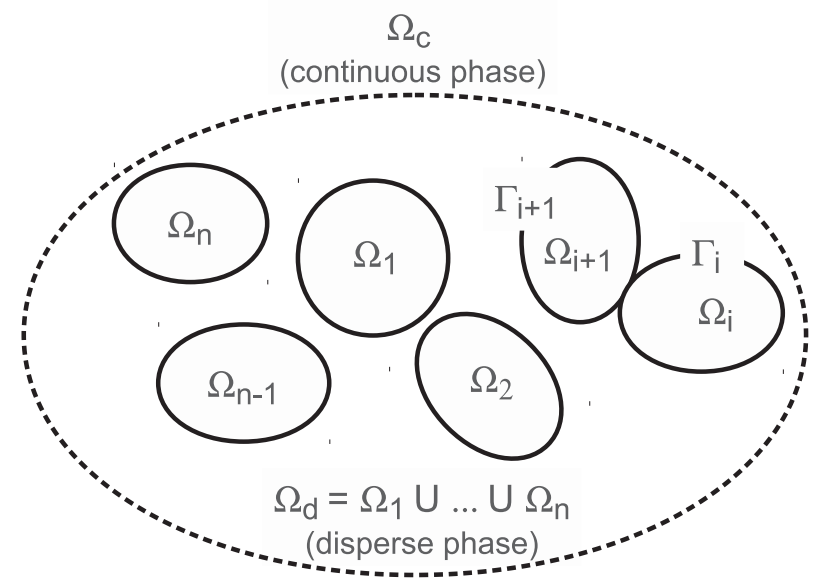

Figure 1: Schematic representation of the continuous fluid phase $\Omega_{c}$ and dispersed fluid phase $\Omega_{d}$. Interfaces are represented by $\Gamma_{i}$.

method (Olsson et al., 2005; Balcázar et al., 2014), hence, mass conservation issue that is known to affect classical level-set formulations is circumvented. Following the work of Olsson et al. (2005), a regularized indicator function, $\phi_{i}$, is used for interface capturing:

$$
\phi_{i}(\mathbf{x}, t)=\frac{1}{2}\left(\tanh \left(\frac{d_{i}(\mathbf{x}, t)}{2 \varepsilon}\right)+1\right) \quad \text { with } i=1, . ., n_{d}
$$

where $d_{i}(\mathbf{x}, t)$ is a signed distance function (Osher et al., 1988; Sussman et al., 1994) and $\varepsilon$ is a tunable parameter that sets the thickness of the interface profile. With this profile the interface, $\Gamma_{i}$, is defined by the location of the $\phi_{i}=0.5$ iso-surface, $\Gamma_{i}=\left\{\mathbf{x} \mid \phi_{i}(\mathbf{x}, t)=0.5\right\}$.

The conservative level-set function $\phi_{i}$ is advected by a vector field $\mathbf{v}$ that is the solution of the Navier-Stokes equations (Eqs. 1 and 2). The interface transport equation can be written in conservative form provided the velocity field is solenoidal, $\nabla \cdot \mathbf{v}=0$, namely,

$$
\frac{\partial \phi_{i}}{\partial t}+\nabla \cdot \phi_{i} \mathbf{v}=0
$$

Furthermore, an additional re-initialization equation (Olsson et al., 2005; Harten , 1977) is introduced to keep the profile and thickness of the interface 
constant,

$$
\frac{\partial \phi_{i}}{\partial \tau}+\nabla \cdot \phi_{i}\left(1-\phi_{i}\right) \mathbf{n}_{i}=\nabla \cdot \varepsilon \nabla \phi_{i}
$$

This equation is advanced in pseudo-time $\tau$, it consists of a compressive term, $\left.\phi_{i}\left(1-\phi_{i}\right) \mathbf{n}_{i}\right|_{\tau=0}$, which forces the level-set function to be compressed onto the interface along the normal vector $\mathbf{n}_{i}$, and of a diffusion term $\nabla \cdot \varepsilon \nabla \phi_{i}$ that ensure the profile remains of characteristic thickness $\varepsilon$. Geometrical information on the interface $\Gamma_{i}$, such as normal vector $\mathbf{n}_{i}$ or curvature $\kappa_{i}$, is obtained through:

$$
\begin{aligned}
\mathbf{n}_{i}\left(\phi_{i}\right) & =\frac{\nabla \phi_{i}}{\left\|\nabla \phi_{i}\right\|} \\
\kappa_{i}\left(\phi_{i}\right) & =-\nabla \cdot \mathbf{n}_{i}
\end{aligned}
$$

The accurate computation of the surface tension $\left(\mathbf{f}_{\sigma}\right.$ in Eq. 1$)$ is one of the most critical elements of any method designed to follow the motion of the interface between immiscible fluids. In this regard, implementing surface tension in a numerical scheme involves two issues: the curvature $\kappa_{i}$ needs to be determined, and the resulting pressure jump must be applied appropriately to the fluids. Because finite-volume method is used for discretization of governing equations, the aforementioned problems can be conveniently addressed through the continuous surface force (CSF) method proposed by Brackbill et al. (1992); which has been extended to include multiple markers in the same grid cell. Thus, the surface tension term, $\mathbf{f}_{\sigma}$ in Eq. 1, is converted to a volume force as follows:

$$
\mathbf{f}_{\sigma}=\sigma \sum_{i} \kappa_{i}\left(\phi_{i}\right) \nabla \phi_{i}
$$

where $\kappa_{i}$ is given by Eq. (8) and $\sigma$ is the surface tension coefficient. In addition, the fluid properties are regularized by employing a global level-set function, $\phi_{d}$, for the disperse phase:

$$
\rho=\rho_{d} \phi_{d}+\rho_{c}\left(1-\phi_{d}\right) \quad \mu=\mu_{d} \phi_{d}+\mu_{c}\left(1-\phi_{d}\right)
$$

where

$$
\phi_{d}(\mathbf{x}, t)=\max \left\{\phi_{1}(\mathbf{x}, t), \ldots, \phi_{n_{d}-1}(\mathbf{x}, t), \phi_{n_{d}}(\mathbf{x}, t)\right\}
$$




\section{Numerical method}

In this section, the numerical method is presented for the sake of completeness. The governing equations have been discretized on a collocated unstructured grid arrangement by means of the finite-volume method, according to Balcázar et al. (2014). In order to avoid unphysical oscillations in the levelset function, a TVD Superbee limiter (Sweby et al., 1984; Balcázar et al., 2014), is used to discretize the convective term in advection Eq. (5), while the compressive term of the re-initialization Eq. (6) is discretized by using a central difference (CD) scheme. A CD scheme is used to discretize the convective term of momentum Eq. (1) unless otherwise stated. A distanceweighted linear interpolation is used to find the cell-face values, while gradients are computed at cell centroids by using the least-squares method (see Balcázar et al. (2014) for details).

The velocity-pressure coupling has been solved by means of a classical fractional step projection method (Chorin , 1968; Guermond et al., 2006). Thus, momentum Eq. (1) is decomposed into two steps:

$$
\frac{\rho \mathbf{v}^{*}-\rho \mathbf{v}^{n}}{\Delta t}=-\frac{3}{2} \mathbf{A}_{h}\left(\rho \mathbf{v}^{n}\right)+\frac{1}{2} \mathbf{A}_{h}\left(\rho \mathbf{v}^{n-1}\right)+\mathbf{D}_{h}\left(\mathbf{v}^{n}\right)+\rho \mathbf{g}+\sigma \sum_{i} \kappa_{i} \nabla_{h}\left(\phi_{i}\right)
$$

and

$$
\mathbf{v}^{n+1}=\mathbf{v}^{*}-\frac{\Delta t}{\rho} \nabla_{h}\left(p^{n+1}\right)
$$

where $\nabla_{h}$ represents the gradient operator, $D_{h}(\mathbf{v})=\nabla_{h} \cdot \mu\left(\nabla_{h} \mathbf{v}+\nabla_{h}^{T} \mathbf{v}\right)$ is the diffusion operator, and $\mathbf{A}_{h}(\rho \mathbf{v})=\nabla_{h} \cdot(\rho \mathbf{v v})$ is the advection operator.

The resulting velocity $\mathbf{v}^{*}$ from Eq. (12), which does not satisfy the continuity Eq. (2), is corrected by Eq. (13). Substituting Eq. (12) into the continuity Eq. (2) yields a Poisson equation for pressure,

$$
\nabla_{h} \cdot\left(\frac{1}{\rho} \nabla_{h}\left(p^{n+1}\right)\right)=\frac{1}{\Delta t} \nabla_{h} \cdot\left(\mathbf{v}^{*}\right)
$$

Poisson Eq. (14) discretization, leads to a linear system, which is solved by using a preconditioned conjugate gradient method. In order to avoid pressure-velocity decoupling when the pressure projection is made on collocated meshes (Rhie and Chow , 1983; Felten and Lund, 2006), a cell-face velocity $\mathbf{v}_{f}$ is defined so that $\nabla_{h} \cdot \mathbf{v}=0$ (see Eq. 2) at each control volume. 
Namely in discretized form:

$$
\mathbf{v}_{f}=\sum_{q \in\{P, F\}} \frac{1}{2}\left(\mathbf{v}_{q}^{n+1}+\frac{\Delta t}{\rho\left(\phi_{q}^{n}\right)}\left(\nabla_{h} p^{n+1}\right)_{q}\right)-\frac{\Delta t}{\rho_{f}}\left(\nabla_{h} p^{n+1}\right)_{f}
$$

where $P$ and $F$ are denoting the adjacent cell nodes to the face $f$ (see Balcázar et al. (2014)). This velocity is used to advect the markers and momentum in Eq. 5 and Eq. 12 respectively. For the temporal discretization, explicit Adams-Bashforth scheme is used to solve the momentum Eq. (12), while for the corrector Eq. (13) an explicit first-order scheme has been used. Interface propagation Eq. (5) and re-initialization Eq. (6) are integrated in time with a 3-step third-order accurate TVD Runge-Kutta method (Gottlieb et al., 1998). Solving Eq. (6) to steady-state results in a smooth transition of $\phi$ at the interface which is a function of the diffusion coefficient $\varepsilon$. In this paper, all numerical simulations were performed by setting $\varepsilon=0.5 h^{0.9}$ where $h=\left(V_{\text {cell }}\right)^{1 / 3}$ is the characteristic size of the grid cell and $V_{\text {cell }}$ is the cell volume. Therefore $\varepsilon$ is chosen to be as small as possible in order to reduce the smearing of: density, viscosity and surface tension force; while the transition in the CLS function, $\phi$, is enough smooth to avoid numerical issues in the solution of the re-initialization Eq. 6. In present simulations two iterations per physical time-step of re-initialization Eq. (6) are sufficient to keep the profile of the level-set function.

The time increment $\Delta t$, which is limited by the CFL conditions and the stability condition for the capillary force, is given by:

$$
\Delta t=0.1 \min \left(\frac{h}{\|\mathbf{v}\|}, \frac{\rho h^{2}}{\mu},\left(\frac{h}{\|\mathbf{g}\|}\right)^{1 / 2}, h^{3 / 2}\left(\frac{\rho_{1}+\rho_{2}}{4 \pi \sigma}\right)^{1 / 2}\right)
$$

Thus, the computational approach for simulating bubbles and drops without numerical coalescence can be summarized as follows:

1. Initialize $\mathbf{v}_{P}(P \equiv$ cell centroid $), \mathbf{v}_{f}(f \equiv$ face centroid $)$, and $\phi_{i}$ for $i=1, . ., n_{d}$.

2. Calculate the time step $(\Delta t)$ according to Eq. 16 .

3. Advection (using $\mathbf{v}_{f}$ ) and re-initialization of $\phi_{i}$ for $i=1, . ., n_{d}$

4. Computation of $\mathbf{n}_{i}, \kappa_{i}$ for $i=1, . ., n_{d}$, and $\phi_{d}, \rho_{d}, \mu_{d}$

5. Computation of $\mathbf{v}_{P}$ and $p$ by solving Eq. 12, Eq. 14 and Eq. 13.

6. Computation of $\mathbf{v}_{f}$ by Eq. 15 . 
7. Repeat steps 2-6 until time step required.

The reader is referred to Balcázar et al. (2014) for technical details on the discretization of the Navier-Stokes/level-set equations on collocated unstructured grids, that are beyond the scope of this paper. The described numerical methods were implemented in an in-house solver called TermoFluids (Lehmkuhl et al., 2007), which is a parallel C++ code designed for Direct Numerical Simulation and Large Eddy Simulation of turbulent flows (Trias and Lehmkhul, 2011; Rodríguez, 2011). Simulations presented in this work were computed on the in-house JFF cluster, which is composed by 40 cluster nodes, each node has 2 AMD Opteron with 16 Cores for each CPU linked with 64 Gigabytes of RAM memory and an infiniband QDR 4X network interconnection between nodes. The number of cores used for the test cases studied in the next section are: 192 cores for the drop impact on a liquid-liquid interface (section 4.1), 128 cores for the binary droplet collision with bouncing outcome (section 4.2), and 256 cores for the gravity-driven bubbly flow in a vertical pipe (section 4.3).

\section{Numerical experiments}

\subsection{Drop impact on a liquid-liquid interface}

The gravity-driven impact of a single drop onto a liquid-liquid interface is studied in order to validate the accuracy of the implemented multiple marker level-set method against experimental and numerical data reported by Mohamed-Kassim et al. (2003) and Coyajee and Boersma (2009), respectively. Following the work of Clift et al. (1978), bubbles and droplets rising or falling freely in infinite media can be characterized by four dimensionless numbers:

$$
M \equiv \frac{g \mu_{c}^{4} \Delta \rho}{\rho_{c}^{2} \sigma^{3}} \quad E o \equiv \frac{g d^{2} \Delta \rho_{c}}{\sigma} \quad \eta_{\rho} \equiv \frac{\rho_{c}}{\rho_{d}} \quad \eta_{\mu} \equiv \frac{\mu_{c}}{\mu_{d}}
$$

where, $\eta_{\rho}$ and $\eta_{\mu}$ are respectively the density and viscosity ratio; $M$ is the Morton number; Eo is the Eötvös number; $\Delta \rho=\left|\rho_{c}-\rho_{d}\right|$, specifies the density difference between the continuous and dispersed fluid phases, and $d$ is the spherical volume equivalent diameter of the droplet. Here, the subscript $d$ denotes the dispersed fluid phase and $c$ the continuous fluid phase. For given fluids, the Eötvös number is a characteristic of the droplet size and $M$ is a parameter representing the viscosity of the continuous fluid phase. 


\begin{tabular}{llllll}
\hline Case & $\rho_{c}\left[\mathrm{~kg} / \mathrm{m}^{3}\right]$ & $\rho_{d}\left[\mathrm{~kg} / \mathrm{m}^{3}\right]$ & $\mu_{c}[\mathrm{mPas}]$ & $\mu_{d}[\mathrm{mPas}]$ & $\sigma[\mathrm{mN} / \mathrm{m}]$ \\
\hline A & 949 & 1128 & 19.0 & 6.3 & 29.1 \\
B & 960 & 1131 & 48.0 & 6.7 & 29.5 \\
\hline
\end{tabular}

Table 1: Material properties in experiments of Mohamed-Kassim et al. (2003). The subscript $d$ is used for silicon oil. The subscript $c$ is used for water/glycerin.

\begin{tabular}{lllll}
\hline Case & $E o$ & $M$ & $\eta_{\rho}$ & $\eta_{\mu}$ \\
\hline A & 6.4 & $8.82 \times 10^{-8}$ & 1.19 & 0.33 \\
B & 6.0 & $1.03 \times 10^{-7}$ & 1.18 & 0.14 \\
\hline
\end{tabular}

Table 2: Dimensionless parameters used in present numerical simulations and experiments of Mohamed-Kassim et al. (2003).

In this section, numerical simulations were performed for a set of dimensionless parameters corresponding to conditions of experiments in Mohamed-Kassim et al. (2003) which are listed in Table 2. Material properties used in experiments of Mohamed-Kassim et al. (2003) are listed in Table 1, namely, water as dispersed phase $(d)$ and silicon oil as continuous phase $(c)$. For the sake of comparison, we also introduce the following dimensionless time, $t^{*}=t / t_{i}$, where $t_{i}=d / U_{i}$ and $U_{i}$ is equivalent to the drop terminal velocity. The dimensional values of $d, U_{i}, t_{i}$ and the corresponding dimensionless parameters used in experiments of Mohamed-Kassim et al. (2003) are listed in Table 3,.

$$
R e \equiv \frac{\rho_{c} U_{i} d}{\mu_{c}} \quad W e \equiv \frac{\rho_{c} d U_{i}^{2}}{\sigma} \quad F r \equiv \frac{\rho_{c} U_{i}^{2}}{\Delta \rho g d}
$$

where $R e$ is the Reynolds number defined based on the impact velocity $U_{i}$, $W e$ is the Weber number and $F r$ is the Froude number. The key difference between the two cases is the value of the continuous fluid viscosity, $\mu_{c}$, in Table 1.

The computational set-up is schematically indicated in Fig. 2a. The

\begin{tabular}{llll}
\hline & Units & Case A & Case B \\
\hline Diameter, $d$ & $\mathrm{~cm}$ & 1.03 & 1.03 \\
Drop impact velocity, $U_{i}$ & $\mathrm{~cm} / \mathrm{s}$ & 13.2 & 9.8 \\
Drop impact time scale, $t_{i}$ & $\mathrm{~ms}$ & 78.0 & 105.0 \\
Reynold number, $\mathrm{Re}$ & & 68.0 & 20.0 \\
Weber number, $W e$ & & 7.0 & 3.8 \\
Froude number, $\mathrm{Fr}$ & & 1.0 & 0.6 \\
\hline
\end{tabular}

Table 3: Experimental parameters in experiments of Mohamed-Kassim et al. (2003). 
(a)

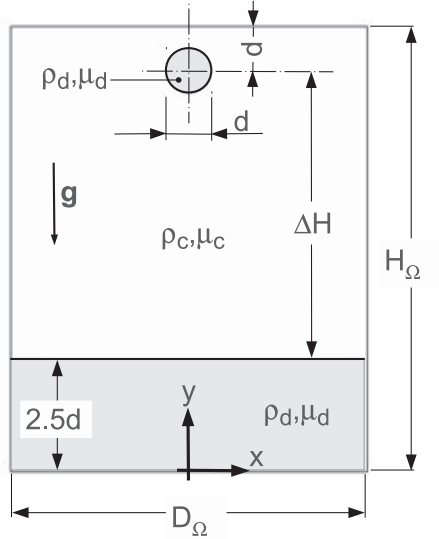

(b)

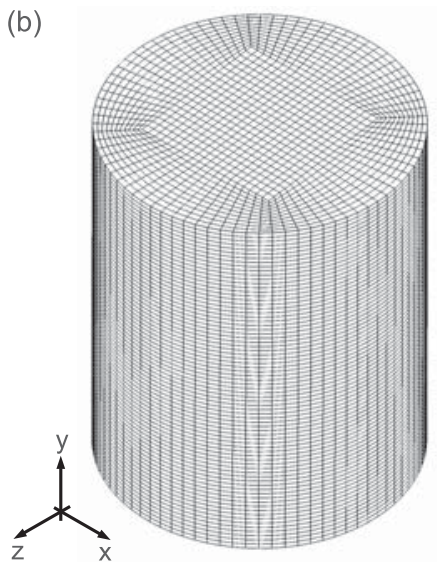

(c)

\begin{tabular}{cccccc}
\hline $\begin{array}{c}\text { Mesh } \\
\text { name }\end{array}$ & Mesh size & $\begin{array}{c}\text { Cells per } \\
\text { plane }\end{array}$ & $\begin{array}{c}\text { Number of } \\
\text { planes }\end{array}$ & $\left(\mathrm{D}_{\Omega}, \mathrm{H}_{\Omega}\right)$ & $\mathrm{h}_{\min }$ \\
\hline $\mathrm{M} 1$ & $1.98 \times 10^{6}$ & 9920 & 200 & $(8 \mathrm{~d}, 10 \mathrm{~d})$ & $\mathrm{d} / 20$ \\
$\mathrm{M} 2$ & $3.60 \times 10^{6}$ & 14400 & 250 & $(8 \mathrm{~d}, 10 \mathrm{~d})$ & $\mathrm{d} / 25$ \\
$\mathrm{M} 3$ & $5.98 \times 10^{6}$ & 19965 & 300 & $(8 \mathrm{~d}, 10 \mathrm{~d})$ & $\mathrm{d} / 30$ \\
\hline
\end{tabular}

Figure 2: (a) Computational setup with initial configuration of the droplet and liquidliquid interface. (b) Mesh configuration. (c) Description of the meshes.

size of the cylindrical domain is fixed to $\left(D_{\Omega}, H_{\Omega}\right)=(8 d, 10 d)$, where $d$ is the initial diameter of the spherical drop, $D_{\Omega}$ is the cylinder diameter and $H_{\Omega}$ is the cylinder height. Thus, confinement effect is minimized, while the drop has enough approach distance to reach terminal velocity before impact. No-slip boundary conditions are imposed at the top $\left(y=H_{\Omega}\right)$ and bottom $(y=0)$ walls, and free-slip boundary condition is used at the lateral boundary, $r=0.5 D_{\Omega}$ (see Fig. 2a). Moreover, with the intend of saving computational resources such as computational time and occupation of memory, our simulations were performed on a non uniform hexahedral mesh, as shown in Fig. 2b. The mesh was generated by a constant step extrusion of a two-dimensional unstructured grid along the symmetry axis of the cylindrical domain, being the step size $H_{\Omega} / N_{\text {planes }}$, where $N_{\text {planes }}$ is the number of planes in which the vertical axis is divided (see Fig. 2b). The mesh was concentrated around the symmetry axis of the domain, where a uniform grid size $\left(h_{\min }\right)$ was fixed, to maximize resolution of the drop. The mesh size grows exponentially to the border, where it reaches a maximum size (see Fig. 2b). 


\begin{tabular}{lllllll}
\hline & & \multicolumn{2}{l}{$\eta_{\mu}=0.33$} & & $\eta_{\mu}=0.14$ & \\
\cline { 3 - 4 } \cline { 5 - 6 }$h$ & Mesh & $\operatorname{Re}_{i}$ & $e_{h}$ & & $R e_{i}$ & $e_{h}$ \\
\hline$d / 30$ & $M_{3}$ & -65.26 & - & & -22.21 & - \\
$d / 25$ & $M_{2}$ & -65.45 & $2.94 \times 10^{-3}$ & & -21.93 & $1.23 \times 10^{-2}$ \\
$d / 20$ & $M_{1}$ & -65.89 & $9.62 \times 10^{-3}$ & & -21.26 & $4.27 \times 10^{-2}$ \\
\hline
\end{tabular}

Table 4: Estimated errors $\left(e_{h}=\left|\left(R e_{i}^{h=d / 30}-R e_{i}^{h}\right)\left(R e_{i}^{h=d / 30}\right)^{-1}\right|\right)$ for the non-dimensional impact velocity $\left(R e_{i}\right)$.

(a)

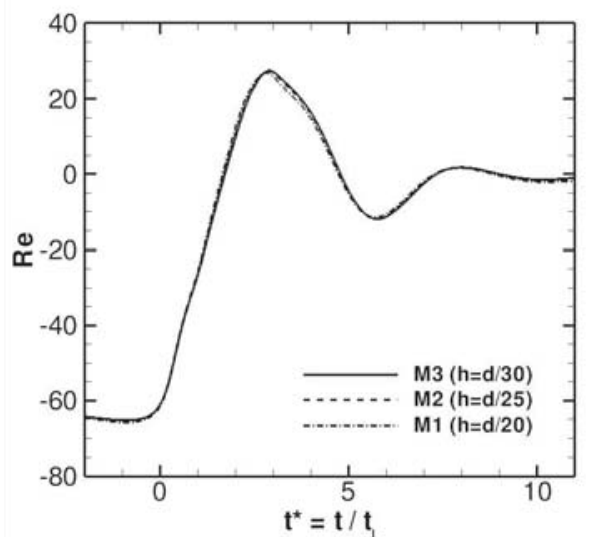

(b)

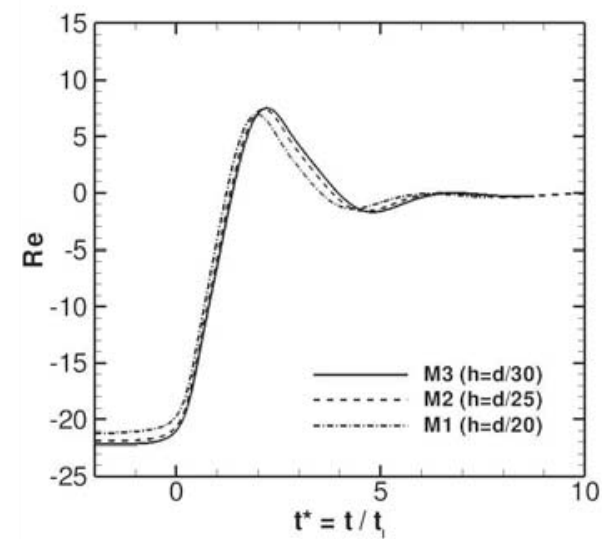

Figure 3: Grid convergence study for the dimensionless droplet velocity, $R e(t)=$ $\rho_{c} d\left(\mathbf{v}_{\text {droplet }}(t) \cdot \mathbf{e}_{z}\right) / \mu_{c}$. Description of the meshes $\left(M_{1}, M_{2}\right.$ and $\left.M_{3}\right)$ is given in Fig. 2c. (a) Case $\eta_{\mu}=0.33$. (b) Case $\eta_{\mu}=0.14$.

Fig. 3 shows the time history of the Reynolds number for different mesh resolutions. The results show essentially the same behavior during the impact event for all resolutions, moreover the estimated errors given in Table 4 show that as the grid size is reduced, the relative difference of nondimensional impact velocity $\left(R e_{i}\right)$ between successive meshes becomes smaller. Therefore, in what follows, the finest mesh resolution $h=d / 30$ is selected for discussion of the results. The computed impact Reynolds number are $R e=65.3$ for the case A with $\eta_{\mu}=0.33$, and $R e=22.2$ for the case B with $\eta_{\mu}=0.14$, which are in close agreement with the experimental values given in Table 3. In Figs. 4a and 4b, the time history of the instantaneous Reynolds number is displayed for different initial approach distances between the droplet centroid and the liquid-liquid interface, $\Delta H$ (see Fig. 2a). These simulations were 
(a)

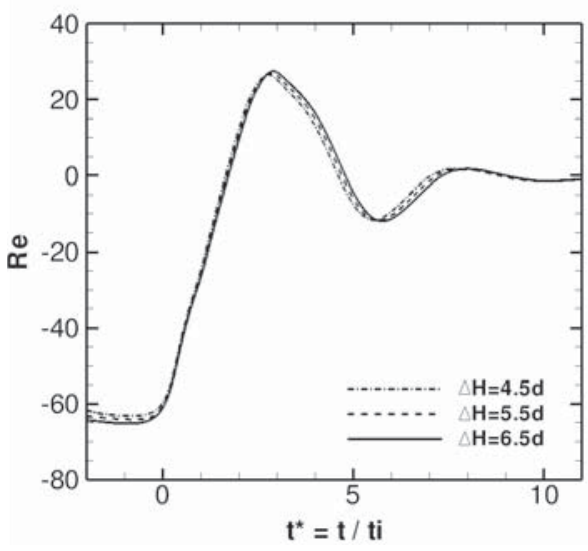

(b)

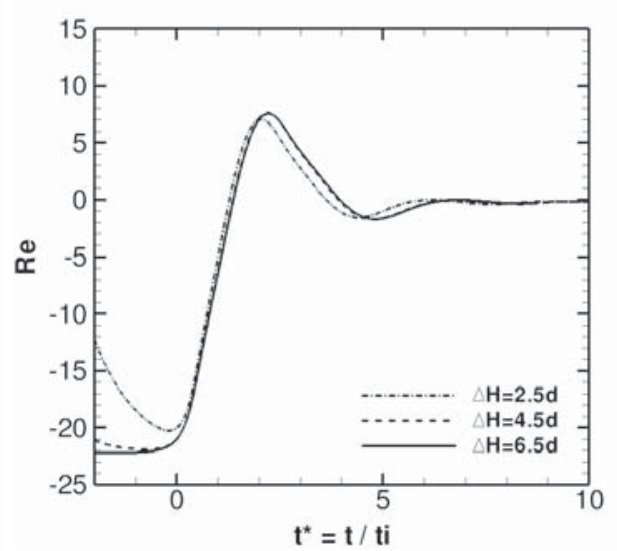

Figure 4: Time evolution of the dimensionless droplet velocity, $R e(t)=\rho_{c} d\left(\mathbf{v}_{\text {droplet }}(t)\right.$. $\left.\mathbf{e}_{z}\right) / \mu_{c}$, as a function of the initial distance between the droplet centroid and the liquidliquid interface $(\Delta H)$. All cases were carried out using the mesh $M_{3}$ (see Fig. 2c). (a) Case A: $\eta_{\mu}=0.33(\mathrm{~b})$ case B: $\eta_{\mu}=0.14$

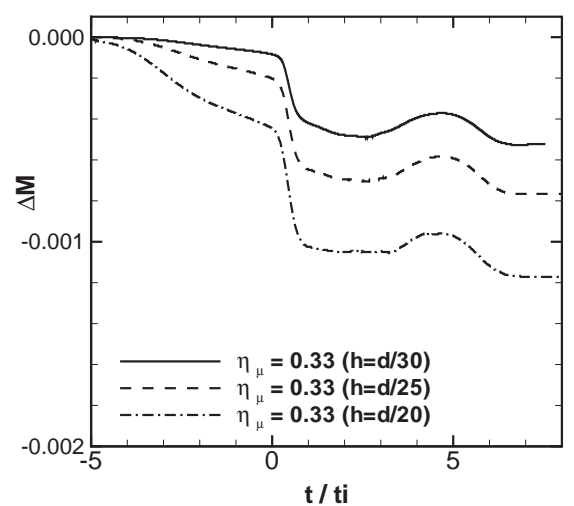

Figure 5: Time evolution of error in mass conservation (related to $\phi_{d}$ ) for the droplet impact on a fluid-fluid interface with $\eta_{\mu}=0.33, \Delta M=(M(t)-M(0)) / M(0)$, with $M(t)=\int_{\Omega} \phi_{d}(\mathbf{x}, t) d V$.

carried out using a mesh resolution $h=d / 30\left(\right.$ mesh $\left.M_{3}\right)$. It is found that the choice $\Delta H=6.5 d$ is enough to reach convergence of the drop velocity, 

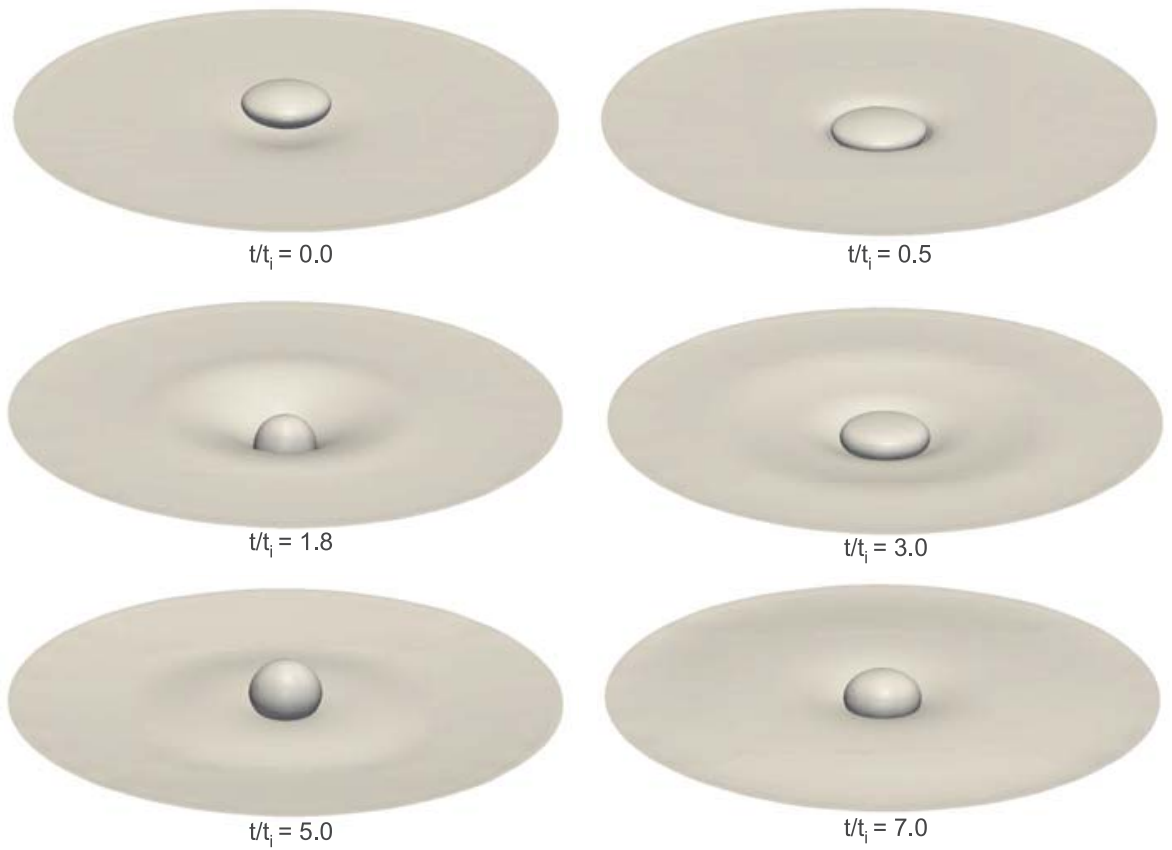

Figure 6: Snapshots of the drop impact onto a liquid-liquid interface, $E_{0}=6.4, M=$ $8.82 \times 10^{-8}, \eta_{\rho}=1.189$ and $\eta_{\mu}=0.33$.

as shown Fig 4a and $4 \mathrm{~b}$. Thus $\Delta H=6.5 d$ is selected for discussion of the numerical experiments.

Figs. 6-7 show a sequence of snapshots of the drop collision for both cases $\eta_{\mu}=0.14$ and $\eta_{\mu}=0.33$ calculated by the multiple marker level-set method; whereas Figs. 8-9 illustrate a comparison of the calculated shape profiles of the drops and the interface $(z=0)$ against experimental images reported by Mohamed-Kassim et al. (2003). Figs. 7a and 8a demonstrate that interface merging occurs automatically when a conventional level-set method (Balcázar et al., 2014) is used, recognizing that the numerical coalescence will lead to correspondingly incorrect surface contours of the droplet/fluidfluid interface compared to those of the experimental images from Figs. 7c and 8c. These results are consistent with numerical simulations performed by Mohamed-Kassim et al. (2004), where a level-set method was combined with a two-dimensional adaptive mesh refinement technique in order to solve the same problem. Moreover, the coalescence time can be delayed for the case 

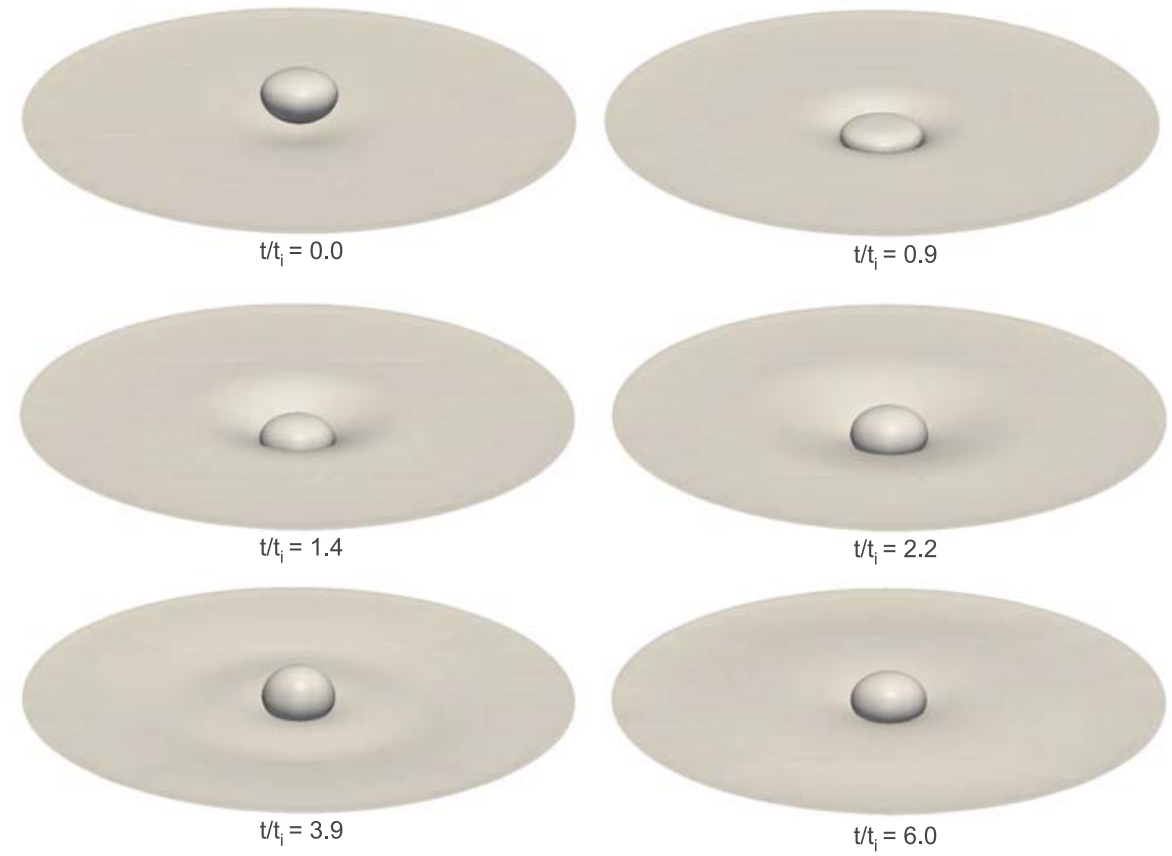

Figure 7: Snapshots of the drop impact onto a liquid-liquid interface, $E o=6.03, M=$ $1.029 \times 10^{-7}, \eta_{\rho}=1.18$ and $\eta_{\mu}=0.14$.

$\eta_{\mu}=0.33$ by increasing local resolution (Zheng et al., 2005; Ceniceros et al. , 2010), however, because of the range of scales as well as the decoupling between impact and coalescence, full three-dimensional simulations are not practical with a conventional level-set method due to the computational expense involved. On the other hand, Figs. $8 \mathrm{~b}$ and $7 \mathrm{~b}$ show that interface profiles calculated by the present multimarker level-set method are in excellent agreement with experiments of Mohamed-Kassim et al. (2003), Figs. 7c and $8 \mathrm{c}$, performed with the same grid resolution. Another important aspect to get accurate simulations is the mass conservation of the different phases. Fig. 5 shows the satisfaction of this requirement, where the mass conservation error is calculated as $\Delta M=(M(t)-M(0)) / M(0)$, with $M(t)=\int_{\Omega} \phi_{d}(\mathbf{x}, t) d V$ and $\phi_{d}$ defined in Eq. 11 as the global level-set marker. When two interfaces are in the same computational cell, mass conservation is affected, however these errors are kept very low, $O\left(10^{-3}\right)$. Moreover, from Fig. 5 it is demonstrated that $\Delta M$ is minimized with mesh refinement. 
(a)
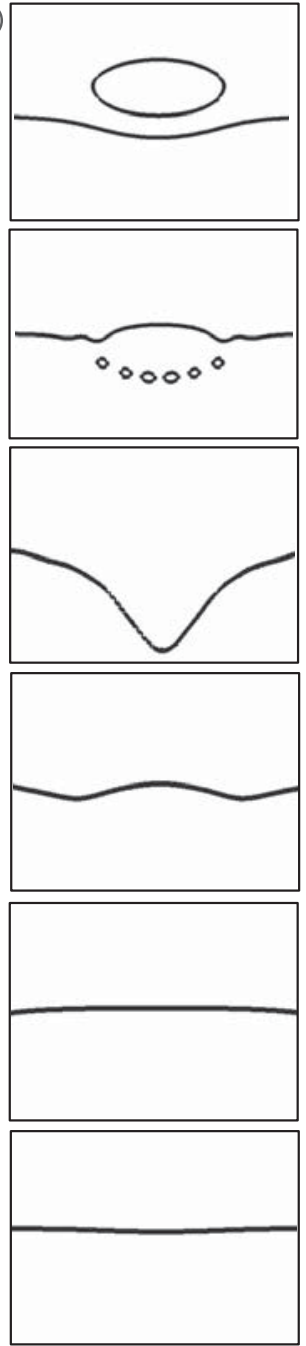

CLS (b)
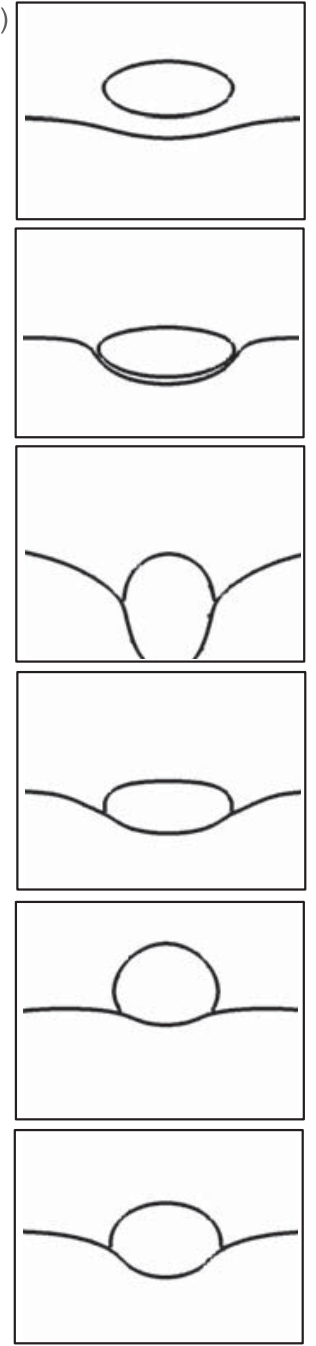

Multiple marker CLS
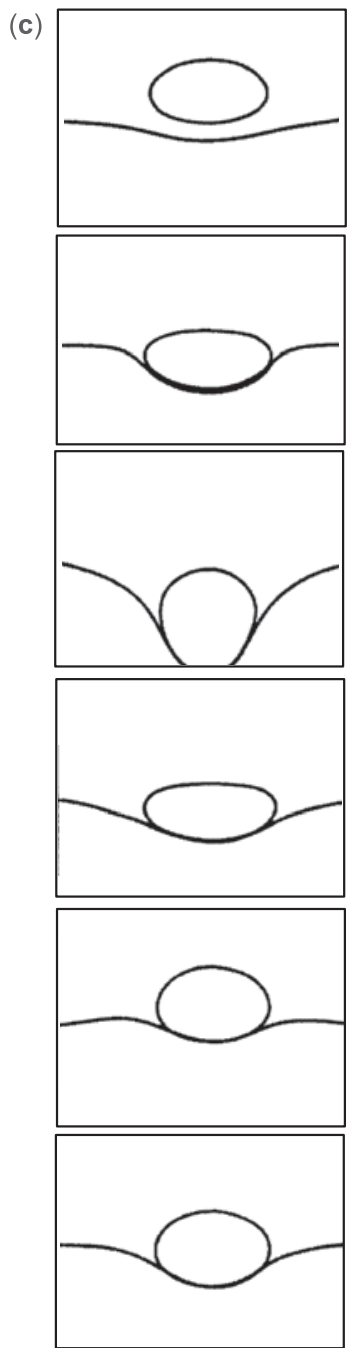

Experiment

Figure 8: Time evolution of the drop shape for $\eta_{\mu}=0.33, t / t_{i}=\{0.0,0.5,1.8,3.0,5.0,7.0\}$ (a) Level-set method (Balcázar et al., 2014). (b) Present computation using the multiple marker level-set method. (c) Experimental findings of Mohamed-Kassim et al. (2003)

Following the work of Mohamed-Kassim et al. (2003), the impact time $t / t_{i}=0$ is defined as the time when the lower drop surface crosses the quiescent liquid-liquid interface level. Thus, prior to the impact, $t / t_{i}<0$ 
(a)
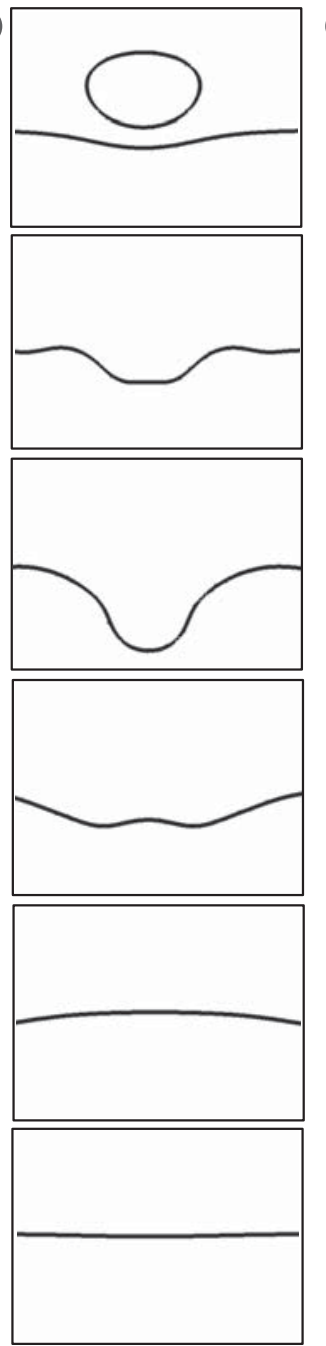

CLS
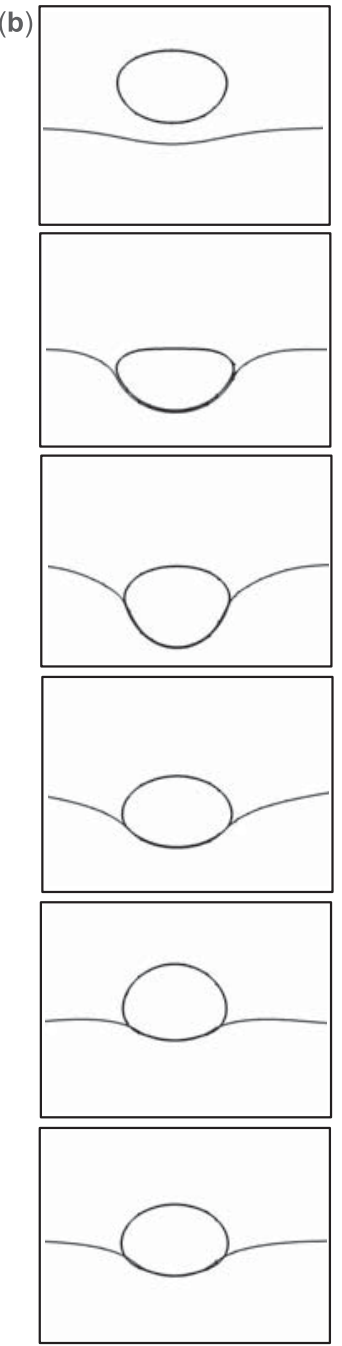

Multiple marker CLS
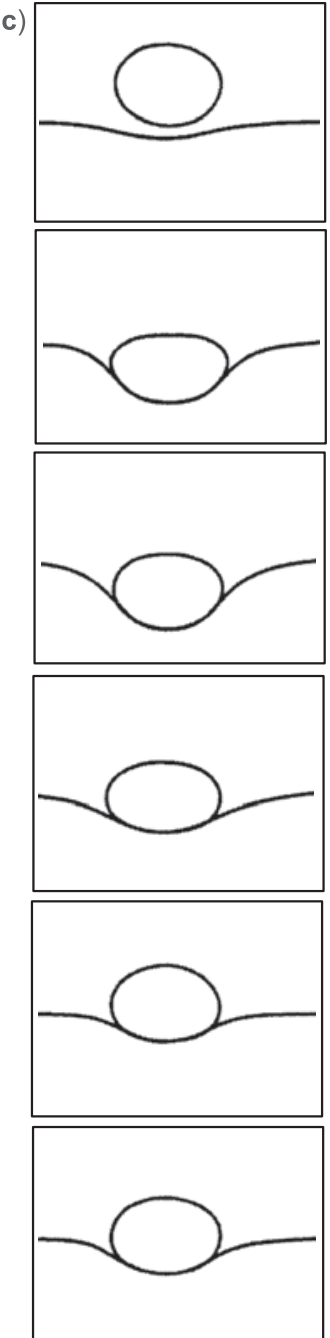

Experiment

Figure 9: Time evolution of the drop shape for $\eta_{\mu}=0.14, t / t_{i}=\{0.0,0.9,1.4,2.2,3.9,6.0\}$ (a) Level-set method (Balcázar et al., 2014). (b) Present computation using the multiple marker level-set method. (c) Experimental findings of Mohamed-Kassim et al. (2003)

(see Fig. 10), both drops have reached steady spheroidal shapes, furthermore, the approach is nearly linear in time until the collision between the drop and liquid-liquid interface is achieved at $t / t_{i}=0$. Additionally, just before the 
(a)

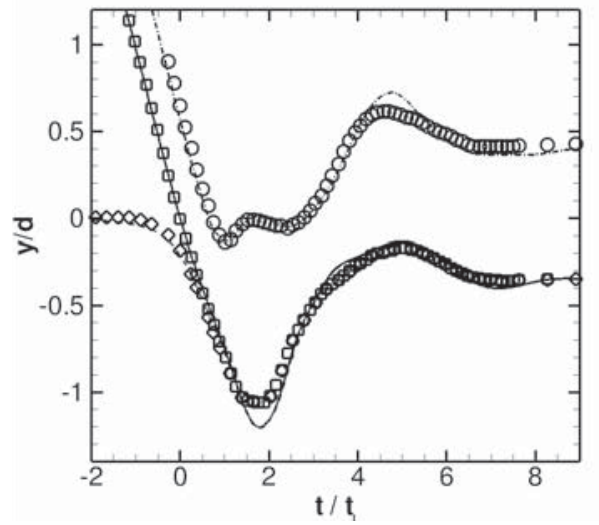

(b)

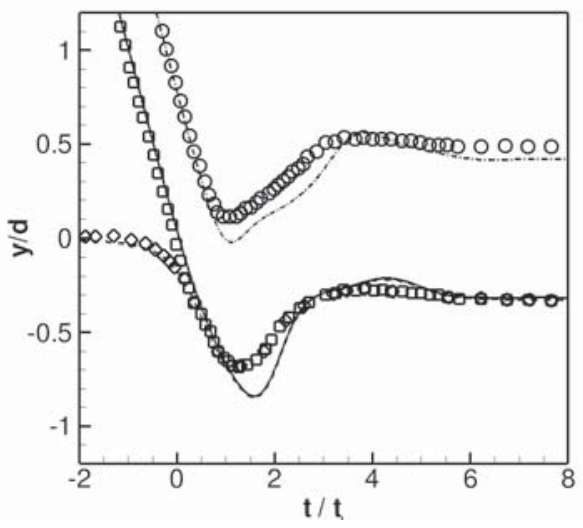

Figure 10: Normalized locations of interface, present results - , lower drop surface $\square$, upper drop surface $\circ$, liquid-liquid surface $\diamond$, (a) $\eta_{\mu}=0.33$ (b) $\eta_{\mu}=0.14$

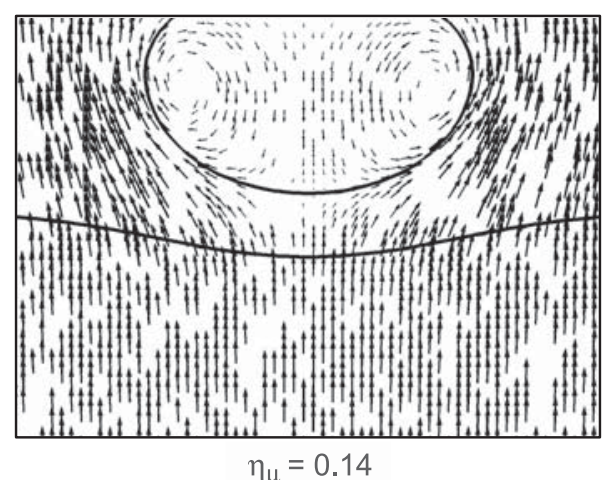

$\eta_{\mu}=0.14$

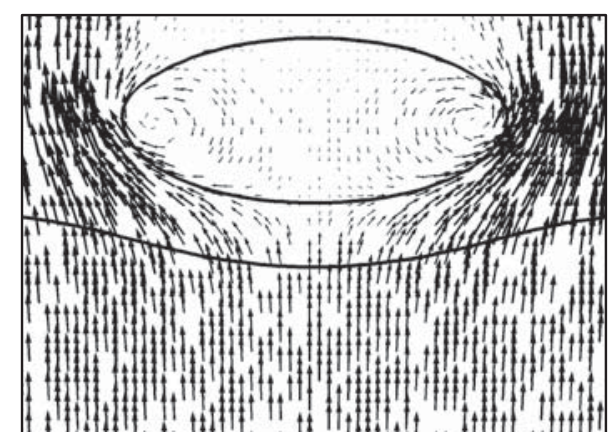

$\eta_{\mu}=0.33$

Figure 11: Present results of the normalized velocity vector field relative to the drop frame of reference at $t / t_{i}=0$

collision, the droplets decelerate and their shapes are flattened, moreover, the trapped fluid near the free surface is drained radially outward, while the gap between the drop and the interface is reduced until a thin fluid layer remains. Indeed, coalescence is inhibited by the thin film of continuous fluid trapped between the droplet and the liquid-liquid interface. In experiments reported by Mohamed-Kassim et al. (2003) a direct analysis of the flow images showed that the film thickness is of order $400 \mu \mathrm{m}$ when the macroscopic steady state is 


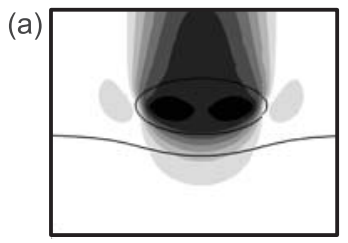

(b)

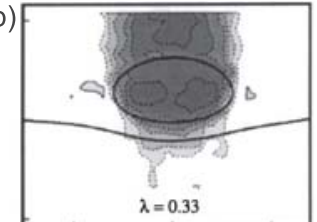

$\mathrm{t} / \mathrm{t}_{\mathrm{i}}=0.0$
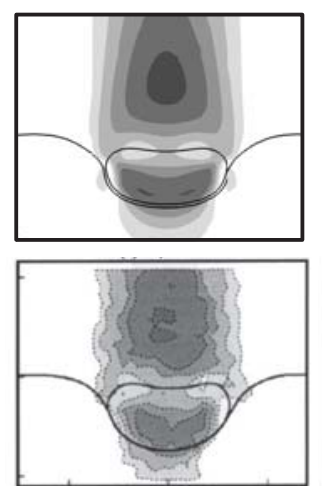

$\mathrm{t} / \mathrm{t}_{\mathrm{i}}=0.9$
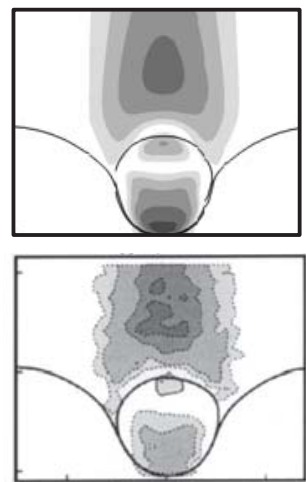

$\mathrm{t} / \mathrm{t}_{\mathrm{i}}=1.4$
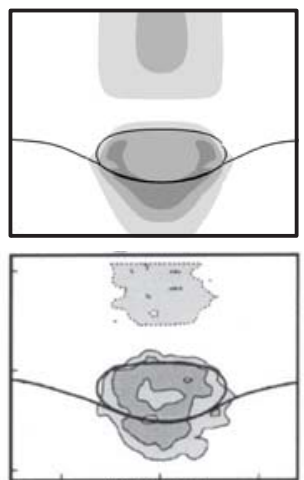

$\mathrm{t} / \mathrm{t}_{\mathrm{i}}=3.0$

$\left|\mathbf{v} \cdot \mathbf{e}_{\mathrm{y}}\right| / \mathrm{U}_{\mathrm{i}} \quad 0.0$

Figure 12: Normalized vertical velocity contour for case $\eta_{\mu}=0.33$. (a) Numerical results using present MLS method. (b) Experimental results given by Mohamed-Kassim et al. (2003).

achieved, which is far smaller than the minimum cell size used in this work. After collision $\left(t / t_{i}>0\right)$, the droplet surfaces are stretched horizontally as they touch the liquid-liquid interface. With time, the remaining inertia inside the drop deflects the interface to a maximum while the drop extends vertically at the same time. This large deformation is followed by a rebound occurring at the time $t / t_{i}=1.4$ and $t / t_{i}=1.8$ for cases $\eta_{\mu}=0.14$ and $\eta_{\mu}=0.33$, respectively. The drop interfaces continue to deform until they reach a steady state, as can be seen in Figs. 8-9. In Fig. 10, normalized centerline locations of the lower drop surface, upper drop surface, and the underlying liquid-liquid interface (aligned with the vertical axis through the drop center) are shown for both viscosity ratios. Numerical results obtained using the present method match fairly well with experimental data reported by Mohamed-Kassim et al. (2003). Similar results were also reported by Coyajee and Boersma (2009).

Fig. 12 and Fig. 13 present contour plots of the normalized vertical velocity, $\left|\mathbf{v} \cdot \mathbf{e}_{y}\right| / U_{i}$, for $\eta_{\mu}=0.33$ and $\eta_{\mu}=0.14$ respectively. A comparison of the present results against experimental findings reported by Mohamed-Kassim et al. (2003) is performed in the aforementioned figures. It can be observed that prior to impact $\left(t / t_{i}=0\right)$ a wake is generated behind each drop, whereas a toroidal shaped region is formed inside them. The dark circles inside the 

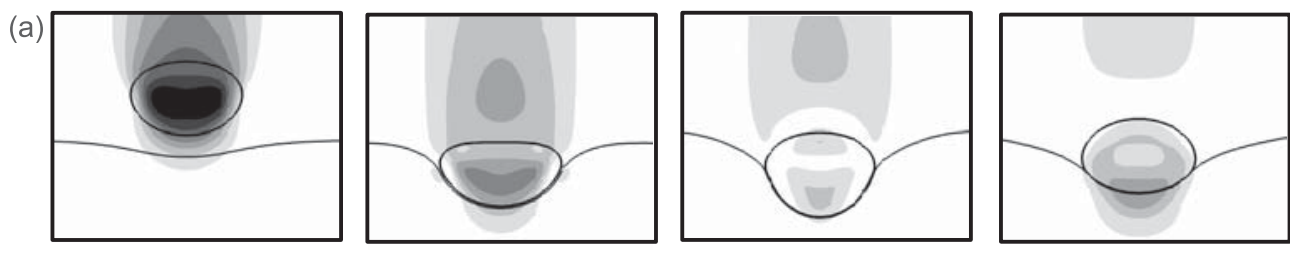

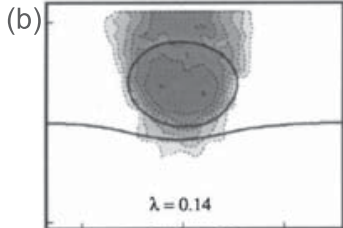

$\mathrm{t} / \mathrm{t}_{\mathrm{i}}=0.0$

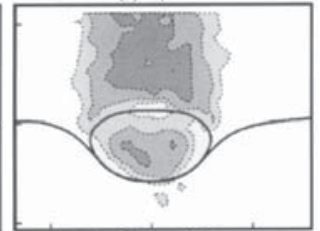

$\mathrm{t} / \mathrm{t}_{\mathrm{i}}=0.9$

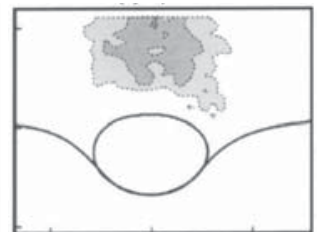

$\mathrm{t} / \mathrm{t}_{\mathrm{i}}=1.4$

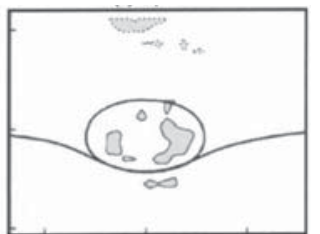

$t / t_{i}=3.0$

$\left|\mathbf{v} \cdot \mathbf{e}_{\mathrm{y}}\right| / \mathrm{U}_{\mathrm{i}} \quad 0.0$

Figure 13: Normalized vertical velocity contour for case $\eta_{\mu}=0.14$. (a) Numerical results using present MLS method. (b) Experimental results given by Mohamed-Kassim et al. (2003).

drops in Figs 12-13 $\left(t / t_{i}=0\right)$ represent regions where the fluid is moving faster, with a maximum downward velocity of approximately $1.2 U_{i}$. The circles mentioned above suggest the existence of a circulation zone, which is clearly confirmed in Fig. 11. Both wakes have similar structure and their width approximates the drop size, however, there is a slower upward velocity in the ambient fluid near the side of the drop for the case $\eta_{\mu}=0.33$ (see Figs. 6-7 at $\left.t / t_{i}=0\right)$. Furthermore, the presence of the interface bellow, causes an upward movement of the ambient fluid around the drops (see Fig. 11). At the time $t / t_{i}=0.9$, although the general structure of the wake is conserved the downward velocities have decreased, moreover a thin film is formed between the drop and the liquid-liquid interface while the drop surface is forced to move radially outward. From Figs. 12-13, it is observed that the aforementioned tendency is maintained as the time advances, and it is more evident for the case where the viscosity ratio is $\eta_{\mu}=0.14$. During the rebound, at $t / t_{i}>1.4$, the surface tension force is dominant (Mohamed-Kassim et al., 2003), moreover, a sudden change of the upper drop flow structure produces an increase in drop height, which is more pronounced in the case with $\eta_{\mu}=0.33$. Finally, at $t / t_{i}>3$, the upward velocity of the drop fluid is higher away from the center than at the center. Thus, the time evolution of wake structure is in close agreement, both qualitatively and quantitatively in 
(a)

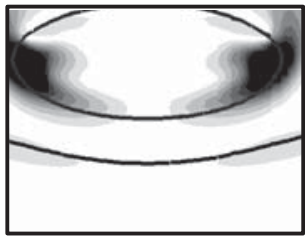

(b)

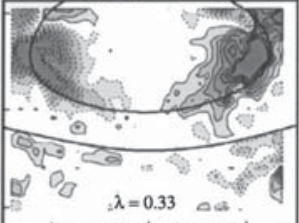

$\mathrm{t} / \mathrm{t}_{\mathrm{i}}=0.0$
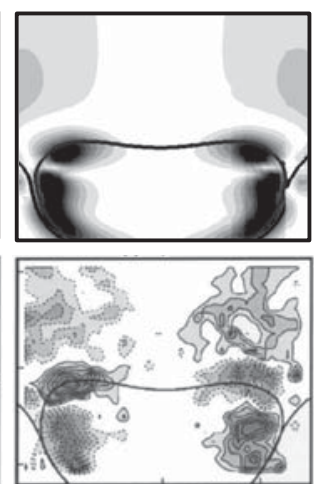

$t / t_{i}=0.9$
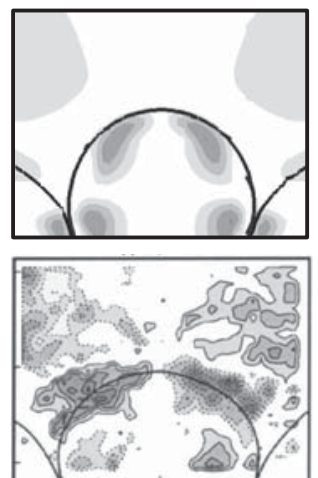

$\mathrm{t} / \mathrm{t}_{\mathrm{i}}=1.4$
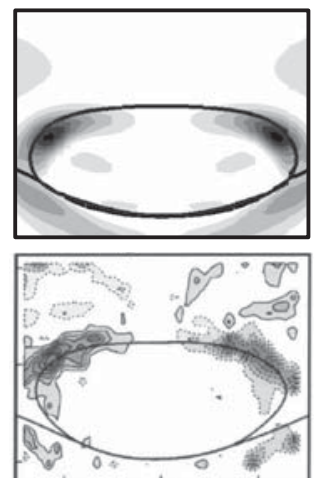

$\mathrm{t} / \mathrm{t}_{\mathrm{i}}=2.2$

$\begin{array}{lll} & \left|\omega_{z}\right| /\left(U_{i} / d\right) & 0.0\end{array}$

Figure 14: Normalized vorticity contour $\left(\omega_{z}\right)$ for case $\eta_{\mu}=0.33$. (a) Numerical results using present MLS method. (b) Experimental results given by Mohamed-Kassim et al. (2003).

comparison with experimental findings reported by (Mohamed-Kassim et al., 2004) and numerical results presented in (Coyajee and Boersma , 2009). The small difference shown in Fig. 10b (case B, $\eta_{\mu}=0.14$ ) between experiment and simulation can be explained due to the impact velocity of the drop is higher in the present numerical simulation $(R e=22.2)$ that in the experiment of Mohamed-Kassim et al. (2003) $(R e=20)$. Furthermore a better performance of the numerical method is observed for the case $\eta_{\mu}=0.33$, which is closer to $\eta_{\mu}=1$, where the simulation does not depend on the continuum approach used to represent the physical properties at the interface. Previous observations are also in close agreement with the numerical findings reported by (Coyajee and Boersma , 2009).

In Figs. 15-14, the normalized vortical contour plots $\left(\omega_{z} /\left(U_{i} / d\right)\right)$ obtained by the present numerical method are contrasted against experimental data reported by Mohamed-Kassim et al. (2003). At the time of impact, $t / t_{i}=0$, two vortices can be observed in the lower half of each drop, which are concentrated near the interface at opposite sides regarding the vertical symmetry axis. At $t / t_{i}=0.9$ the magnitude of the vorticity have decreased, moreover, a new pair of vortices can be identified for both cases, which are localized between the drop interfaces and their wakes. The interaction between the above mentioned vortices reduces the drop internal circulation as 
(a)
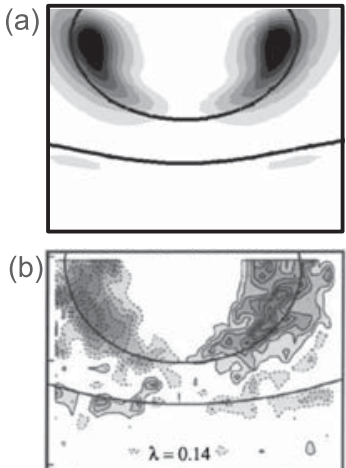

$\mathrm{t} / \mathrm{t}_{\mathrm{i}}=0.0$
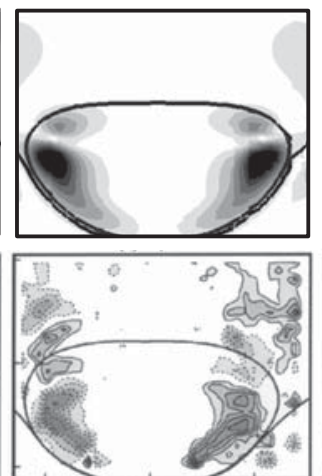

$\mathrm{t} / \mathrm{t}_{\mathrm{i}}=0.9$
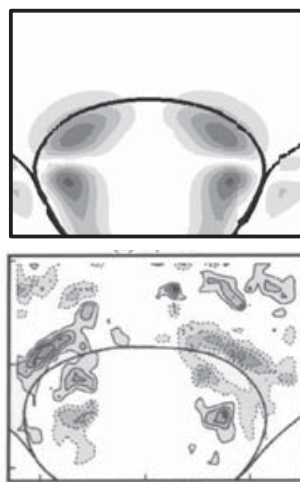

$\mathrm{t} / \mathrm{t}_{\mathrm{i}}=1.4$
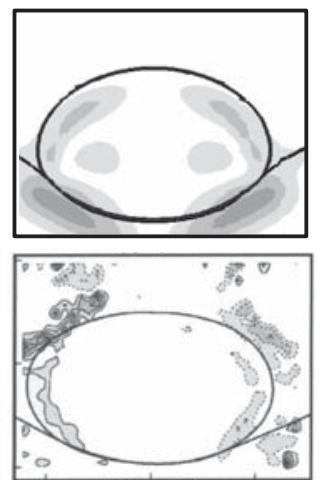

$\mathrm{t} / \mathrm{t}_{\mathrm{i}}=2.2$

$\left|\omega_{\mathrm{z}}\right| /\left(\mathrm{U}_{\mathrm{i}} / \mathrm{d}\right)$ 0.0

Figure 15: Normalized vorticity contour $\left(\omega_{z}\right)$ for case $\eta_{\mu}=0.14$. (a) Numerical results using present MLS method. (b) Experimental results given by Mohamed-Kassim et al. (2003).

the time advances, to finally achieve a rest state (see Figs. 15-14). Thus, regarding the vorticity, present results are also in close agreement with experiments of Mohamed-Kassim et al. (2003) and numerical simulations reported by Coyajee and Boersma (2009).

\subsection{Binary droplet collision with bouncing outcome}

Following experimental studies on binary droplet collision performed by Qian and Law (1997), there are four regimes of distinctively different outcomes for hydrocarbon droplets: (I) permanent coalescence after minor droplet deformation, (II) bouncing, (III) permanent coalescence after major droplet deformation, and (IV) coalescence followed by separation and the concomitant production of satellite droplets, as shown in Fig. 16. The physical criterion that determines the outcome of the collision (coalescence or bouncing) is the gap size or the trapped gas layer thickness between the droplets, therefore, coalescence may happen when the gas layer thickness reaches a critical value which is within the range of the molecular interaction, where the attractive van der Waals force gradually dominates over other forces and eventually pulls the two interfaces together, otherwise the droplets will bounce. Bouncing regime is difficult to handle with classical interface tracking methods (e.g. level-set, volume-of-fluid), because two interfaces will merge automati- 


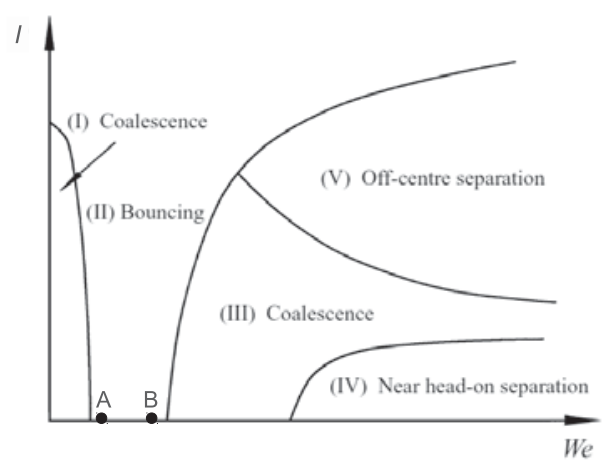

Figure 16: Schematic of various collision regimes of hydrocarbon droplets in 1 atm. air, where $I$ is the dimensionless impact parameter (Eq. 21) and $W e$ is the Weber number (Eq. 19). This figure is reproduced from Qian and Law (1997). Simulated cases are indicated with capitals.

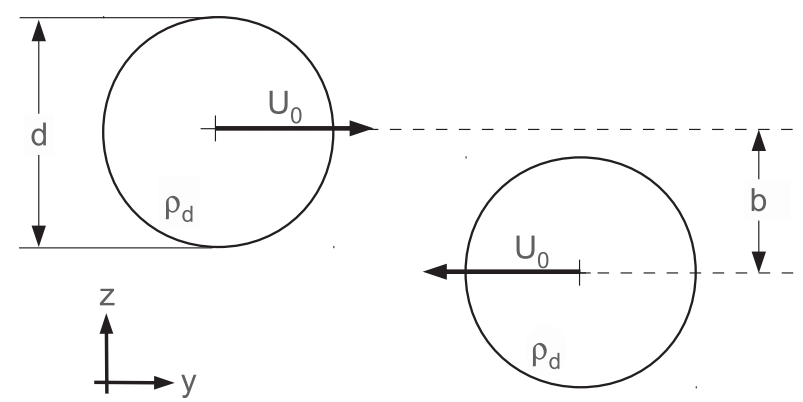

Figure 17: Computational domain and initial conditions. The drops are initially 1.4d apart. 


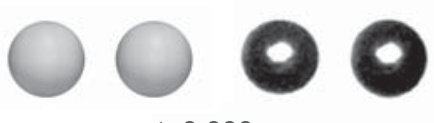

$\mathrm{t}=0.000 \mathrm{~ms}$
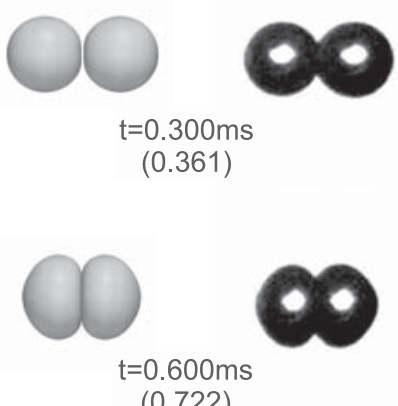

$(0.722)$
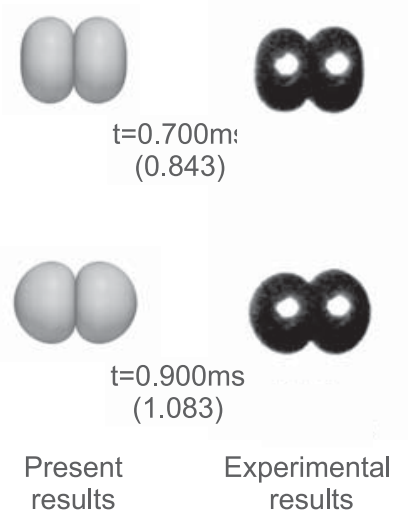
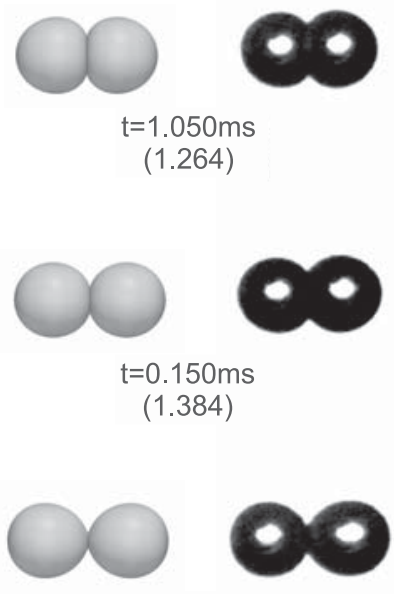

$\mathrm{t}=1.250 \mathrm{~ms}$

(1.505)

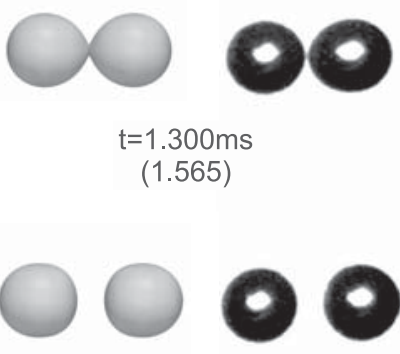

$\mathrm{t}=1.500 \mathrm{~ms}$ (1.806)

Present Experimental

Figure 18: Comparison of bouncing collision sequence between present numerical simulation using multi-marker/level-set method and experimental results reported by Pan et al. (2008). Conditions for case $A$ in Table 5: Tetradecane in 1 atm. air, $d / 2=170.6 \mu m$, $W e=2.27, R e=122.2$. The dimensionless time, $t^{*}=\frac{t}{2 \pi\left(\rho_{d}(d / 2)^{3} / 8 \sigma\right)^{1 / 2}}$, is indicated in parentheses.

cally whenever they move into adjacent cells. Jiang and James (2007) and Li and Fritsching (2011) have studied the droplet collision using a volumeof-fluid method by setting a zero volume-fraction boundary condition on the symmetry plane. In their work, only one droplet, moving toward the sym- 

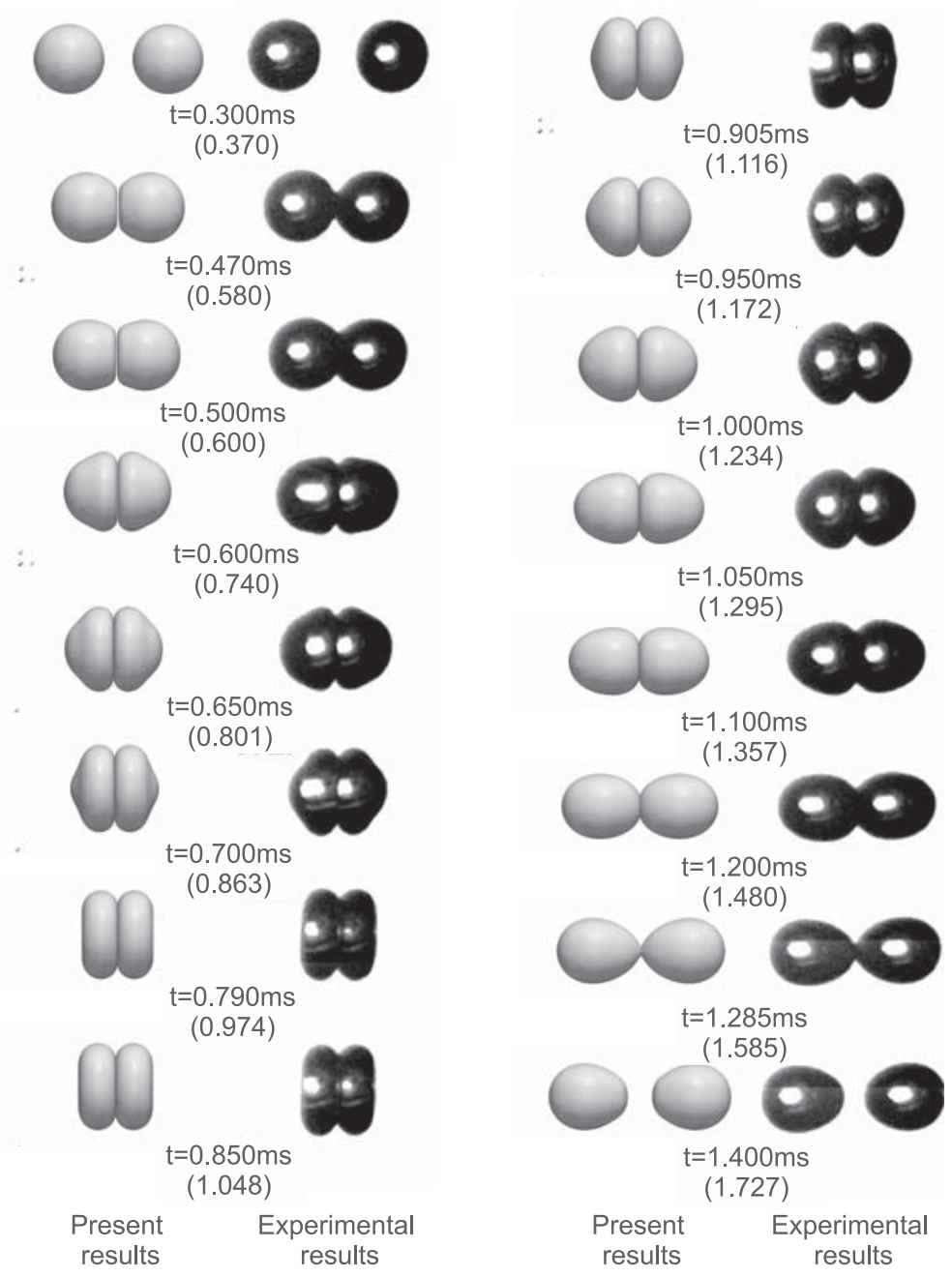

Figure 19: Comparison of bouncing collision sequence between present numerical simulation using multi-marker/level-set method and experimental results reported by Pan et al. (2008). Conditions for case $B$ in Table 5: Tetradecane in 1 atm. air, $d / 2=167.6 \mu m$, $W e=9.33, R e=116.2$. The dimensionless time, $t^{*}=\frac{t}{2 \pi\left(\rho_{d}(d / 2)^{3} / 8 \sigma\right)^{1 / 2}}$, is indicated in parentheses.

metry plane, was tracked and the zero volume-fraction boundary condition was applied using the ghost cells outside the physical boundaries, avoiding the merge of the droplet with their image. On the other hand Nobari et al. 

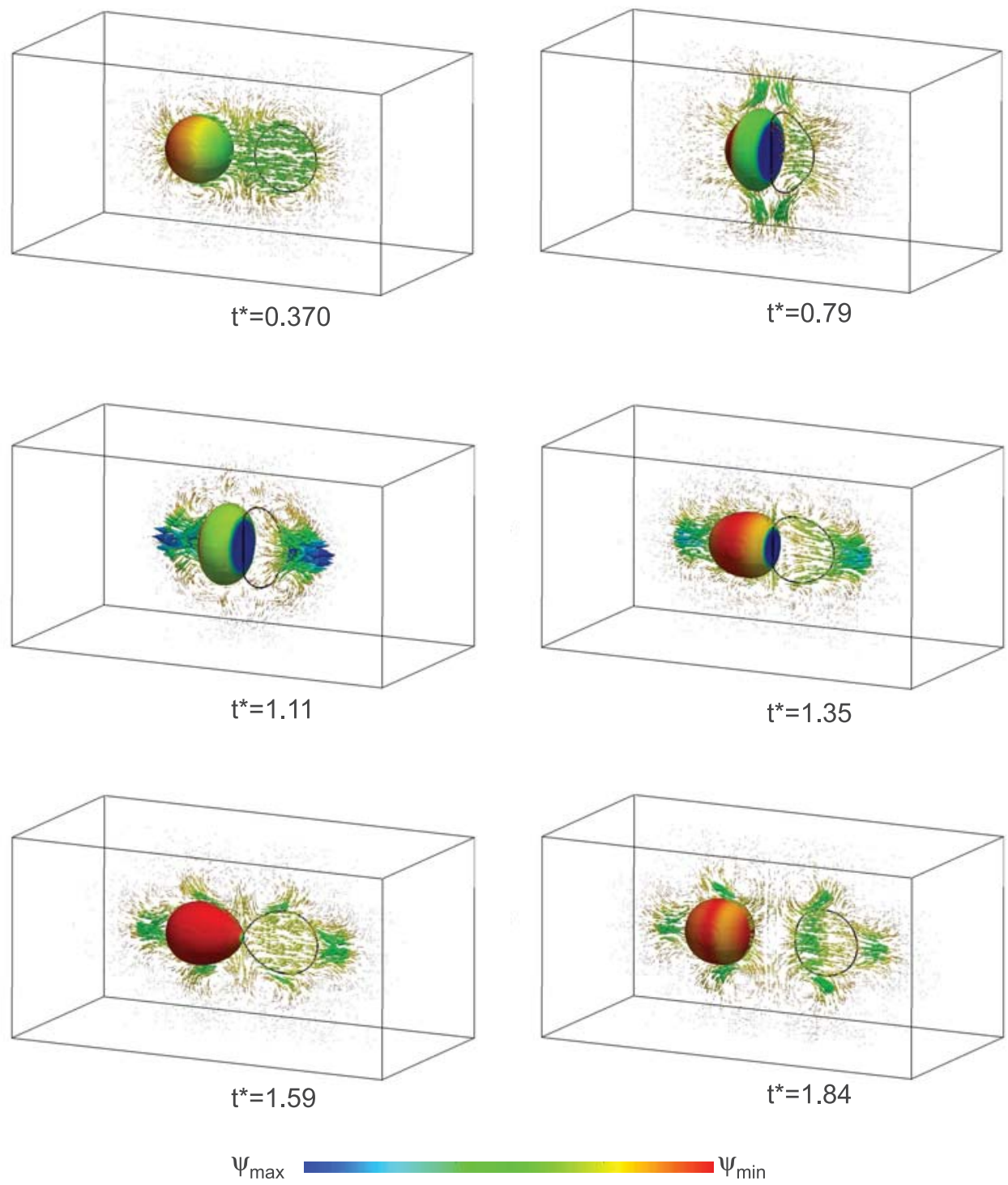

Figure 20: Velocity field and pressure evolution for case $B$ (see Table 5 and Fig. 16). Here $\psi \in\{\|\mathbf{v}\|, p\}$. Pressure field is represented on the droplet surface.

(1996) and Nobari and Tryggvason (1996) used an interface tracking/finite difference technique to simulate the head-on collision of drops with bounc- 


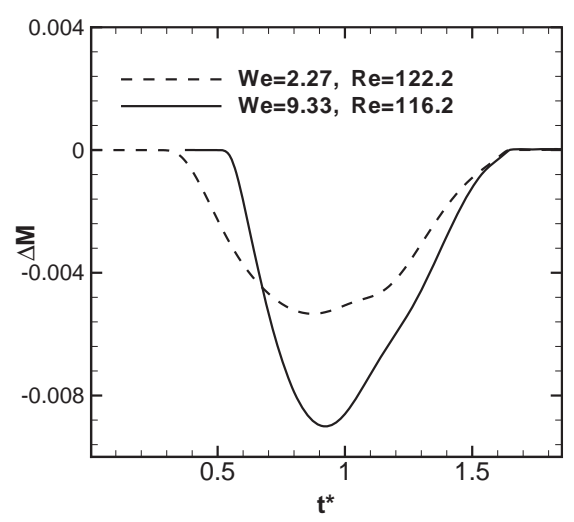

Figure 21: Time evolution of error in mass conservation for the binary droplet collision, $\Delta M=(M(t)-M(0)) / M(0)$, with $M(t)=\int_{\Omega} \phi_{d}(\mathbf{x}, t) d V$.

ing outcome. Recently Pan et al. (2008) studied the dynamics of head-on collision $(I=0)$ between two identical droplets. They performed experiments and numerical simulations based on the front tracking method, with emphasis on the transitions from merging to bouncing and to merging again, as the Weber number was increased. Kwakkel et al. (2013) have also performed numerical simulations of droplet-laden flows using a coupled volumeof-fluid/level-set method with a coalescence/breakup model. Their numerical experiments reproduced experimental results reported by Qian and Law (1997) and Pan et al. (2008). Thus, with the aim of validating the present multi-marker/level-set method, this section is devoted to perform numerical simulations of head-on collisions between equal sized droplets, with bouncing outcome. Furthermore, numerical results are compared with experimental and numerical data reported by Pan et al. (2008) and Kwakkel et al. (2013).

The kinetic and geometric parameters determining the binary droplet collision are shown in Fig. 17. The physical parameters of the disperse liquid phase are $\rho_{d}, \mu_{d}$ and $\sigma$. The size of the colliding droplets is $d$, the relative velocity $2 U_{0}$, and the impact parameter $b$. Nondimensionalization gives a Weber number

$$
W e=\frac{\rho_{d} d U_{0}^{2}}{\sigma}
$$


the Reynolds number

$$
R e=\frac{\rho_{d} U_{0} d}{\mu_{d}}
$$

and the dimensionless impact parameter

$$
I=\frac{b}{d}
$$

where $b$ is the perpendicular distance between the lines that the droplets move along before collision (see Fig. 17). In addition, the density ratio $\eta_{\rho}$ and the viscosity ratio $\eta_{\mu}$ must be specified. Here, the subscript $d$ denotes the fluid in the drop (see Fig. 17).

The computational domain is a rectangular box with size $3 d \times 6 d \times 3 d$ where the largest dimension is along the direction of the collision $(y-a x i s)$. Free slip boundary conditions are used on all six sides of the domain. Initially the distance between the droplet centers is $1.4 d$ (see Fig. 17). An initial velocity field is used, which imposes a uniform velocity of $U_{0}$ only on the droplets in opposite directions without any driving force. These conditions were also used by Pan et al. (2008) and Kwakkel et al. (2013). A uniform cartesian grid $150 \times 300 \times 150$ is used, therefore, the droplet diameter $d$ is resolved by 50 grid points of dimension $h$. The dimensionless properties and geometrical parameters used to perform the numerical experiments are summarized in Table 6. Such selection of dimensionless parameters corresponds to physical properties of tetradecane droplets in atmospheric air, as shown in Table 6. For the sake of comparison with data reported by Pan et al. (2008), a dimensionless time $t^{*}$ is defined as:

$$
t^{*}=\frac{t}{2 \pi\left(\rho_{d}(d / 2)^{3} / 8 \sigma\right)^{1 / 2}}
$$

where $2 \pi\left(\rho_{d}(d / 2)^{3} / 8 \sigma\right)^{1 / 2}$ is the droplet oscillation period, based on inviscid droplet oscillation with small amplitude (Lamb , 1932). In addition, for this particular test case, using a CD scheme to discretize the convective term of the momentum equation (Eq. 1) leads to numerical instabilities. Therefore, regarding the discretization of the convective term a SUPERBEE limiter scheme has been employed, according to the work of Balcázar et al. (2014).

Figs. 18 and 19 show a comparison of the present results against experiments reported by Pan et al. (2008). For the parameters here selected the droplets bounce off without coalescence. The droplet sequences illustrate 


\begin{tabular}{llllll}
\hline Case & $W e$ & $R e$ & $I$ & $\eta_{\rho}$ & $\eta_{\mu}$ \\
\hline $\mathrm{A}$ & 2.27 & 122.2 & 0.0 & 622 & 116.5 \\
$\mathrm{~B}$ & 9.33 & 116.2 & 0.0 & 622 & 116.5 \\
\hline
\end{tabular}

Table 5: Dimensionless parameters for the collision of tetradecane droplets in atmospheric air.

\begin{tabular}{lll}
\hline Air density & $\rho_{c}$ & $1.225 \mathrm{kgm}^{-3}$ \\
Air viscosity & $\mu_{c}$ & $1.827 \times 10^{-5} \mathrm{Nsm}^{-2}$ \\
Tetradecane density & $\rho_{d}$ & $762 \mathrm{kgm}^{-3}$ \\
Tetradecane viscosity & $\mu_{d}$ & $2.128 \times 10^{-3} \mathrm{Nsm}^{-2}$ \\
Surface tension & $\sigma$ & $2.65 \times 10^{-2} \mathrm{Nm}^{-1}$ \\
\hline
\end{tabular}

Table 6: Physical properties for the collision of tetradecane droplets in atmospheric air.

the bouncing collision with increasing Weber number, moreover, the numerical simulations performed by multi-marker/level-set method compare fairly well in terms of the droplet shape and phase. Similar results were reported by Kwakkel et al. (2013) using a coupled volume-of-fluid/level-set method. Figure 20 shows the pressure and the velocity field as they advance in time. As the drops approach each other the pressure between them builds up, leading to the formation of a dimple (see Fig. 20, $t^{*}=0.79$ ). As consequence, the droplets become flatter and the ambient fluid between them is pushed away leaving a thin film of fluid between the droplets, and conversion of the droplet kinetic energy into surface tension energy. The kinetic energy is also partly dissipated through the internal motion within the droplet and, to a much smaller extent, the gas flow inside the gap. Due to the large pressure build up, the drops rebound (see Fig. 20, $t^{*}=1.11$ ) and the film resumes its dimpled shape until the drops are almost round (see Fig. 20, $t^{*}=1.59$ ). Regarding the mass conservation error, Fig. ?? shows the time evolution of $\Delta M(t)=\left(\int\left(\phi_{d}(\mathbf{x}, t)-\phi_{d}(\mathbf{x}, 0)\right) d V\right)\left(\int \phi_{d}(\mathbf{x}, 0) d V\right)^{-1}$, with a maximum fluctuation $O\left(10^{-3}\right)$ during the collision period of the droplets, while the error tends to zero when the interfaces are separated from each other. The above results therefore support the adequacy and accuracy of the computational simulation of binary collision with bouncing outcome.

\subsection{Gravity-driven bubbly flow in a vertical pipe}

This section is devoted to explore the gravity-driven bubbly flow in a vertical pipe. The objective of this test-case is to demonstrate the capability of the presented numerical method to perform long-time simulation 
of bubbly flow in a confined domain without numerical coalescence. Despite simulations of homogeneous bubbly flows have been reported in the past using the front tracking method or Lattice-Boltzmann method (e.g. Bunner and Tryggvason (2002),Bunner and Tryggvason (2003), Esmaeeli and Tryggvason (2005), Yin and Koch (2008)), this is the first time that a multiple marker approach is used in the context of the conservative level-set method to perform such simulations.

(a)

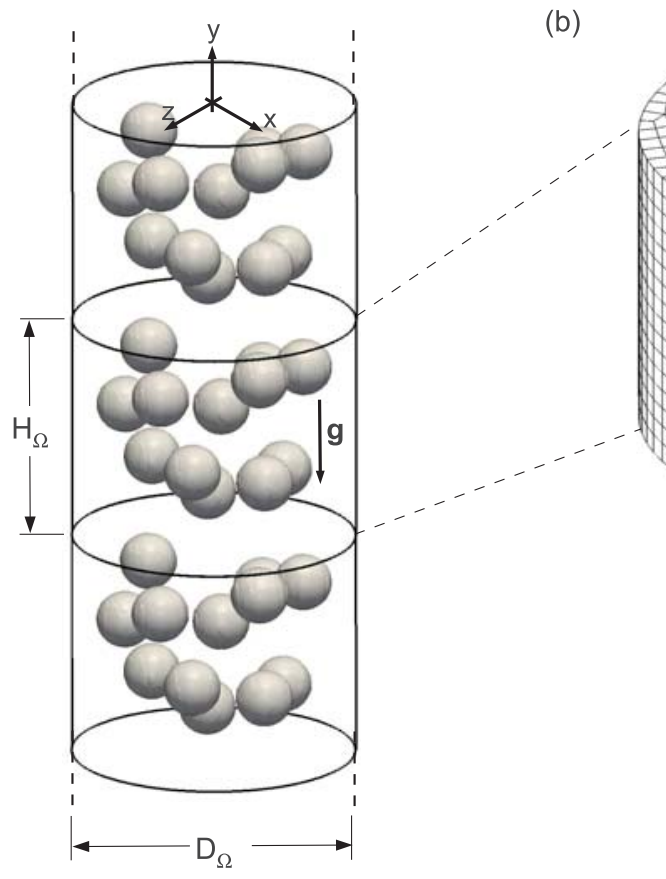

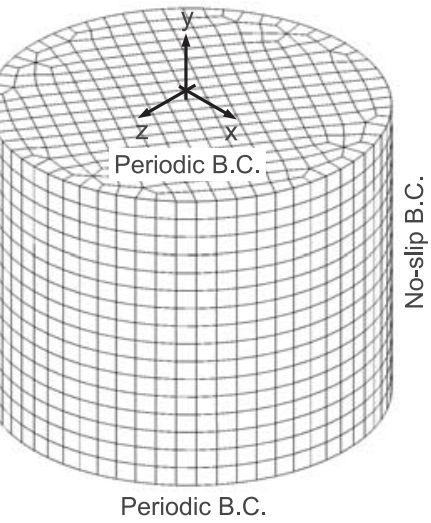

Figure 22: Monodisperse gravity-driven bubbly flow. (a) Computational setup and initial condition. Free bubble array. (b) Mesh configuration (hexahedral cells) and boundary conditions.

In this work, the computational set-up is specified as a vertical cylinder bounded by a rigid wall, with gravity in the $-y$ direction. The size of the domain is $\left(D_{\Omega}, H_{\Omega}\right)=(5 d, 4 d)$, where $d$ is the initial bubble diameter, $D_{\Omega}$ is the cylinder diameter and $H_{\Omega}$ is the cylinder height, as shown in Fig. 22a. Imposed boundary conditions are non-slip at the rigid wall and periodic on the streamwise (y-direction). In this way bubbles go out of the domain on the top side, and they come back in the domain again from the opposite side. 
(a)

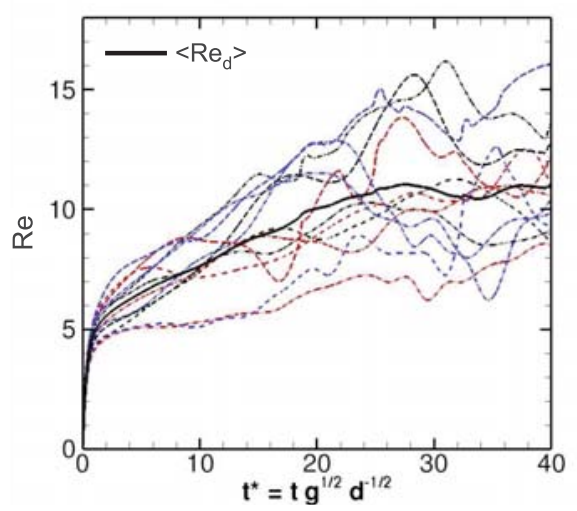

(c)

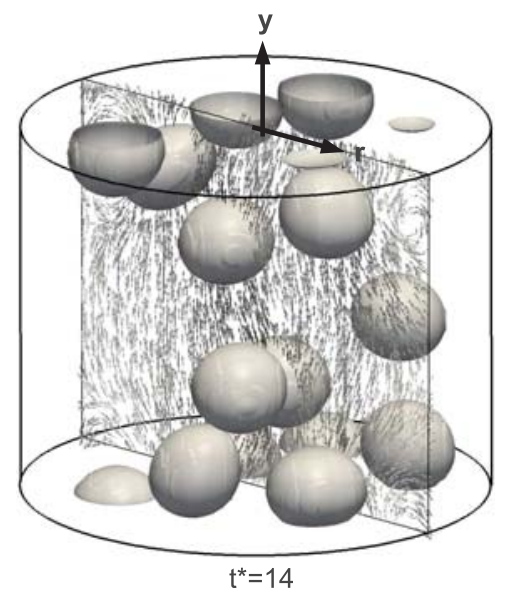

(b)
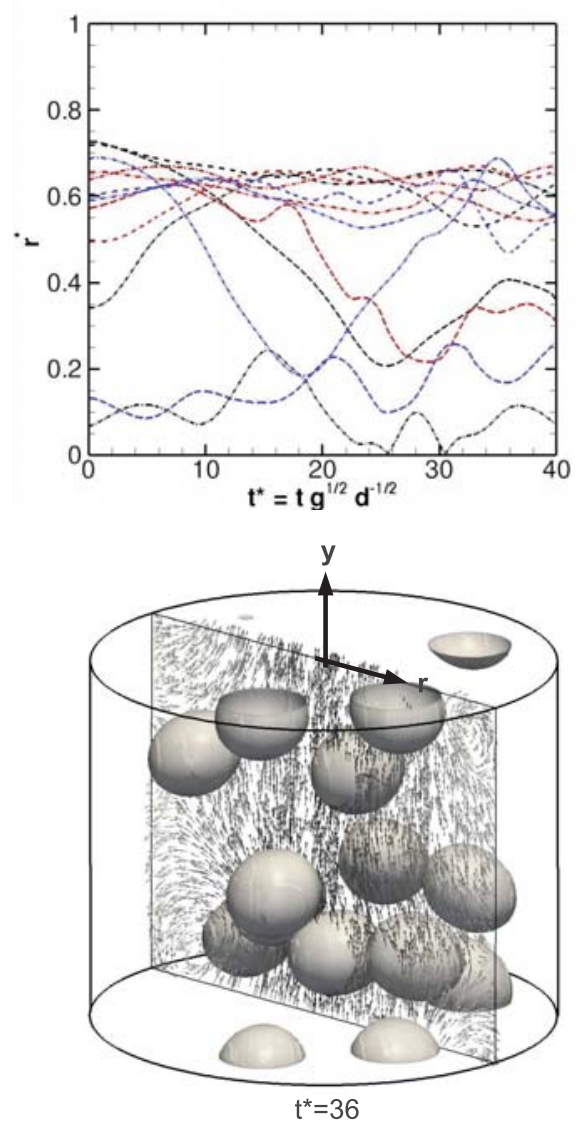

Figure 23: Homogeneous bubbly flow at $E o=3, M=1 \times 10^{-3}, \eta_{\rho}=10$ and $\eta_{\mu}=10$. (a) Time evolution of Reynolds number given by Eq. 25 and 26. (b) Time evolution of the dimensionless radial distance for each bubble, $\left(r^{*}=2\left(x_{i}^{2}+z_{i}^{2}\right)^{1 / 2} D_{\Omega}^{-1}\right)$, hence, $r^{*}=0$ is on the symmetry axis of the cylindrical domain and $r^{*}=1$ is on the cylinder wall. (c) Instantaneous bubble distribution and velocity field.

The mesh was generated by a constant step extrusion of a two-dimensional unstructured grid of quadrilateral cells along the symmetry axis of the cylindrical domain, being the step size $H_{\Omega} / N_{\text {planes }}$, where $N_{\text {planes }}$ is the number of planes in which the vertical axis is divided; therefore, the tridimensional mesh is conformed by hexahedral volumes as shown in Fig. 22b and Table 7.

The gravity-driven bubbly flow is simulated in a vertical column with 


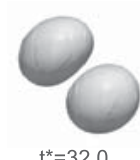

$t^{*}=32.0$

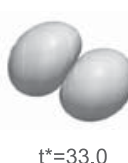

$t^{*}=33.0$

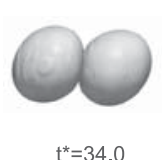

$t^{*}=34.0$

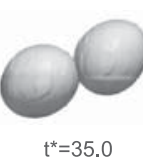

$t^{*}=35.0$

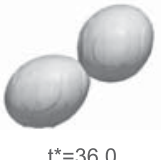

$\mathrm{t}^{*}=36.0$

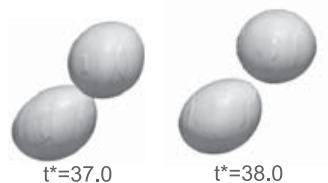

$t^{*}=38.0$

Figure 24: Two bubbles from the $E o=3, M=1 \times 10^{-3}, \eta_{\rho}=10$ and $\eta_{\mu}=10$.

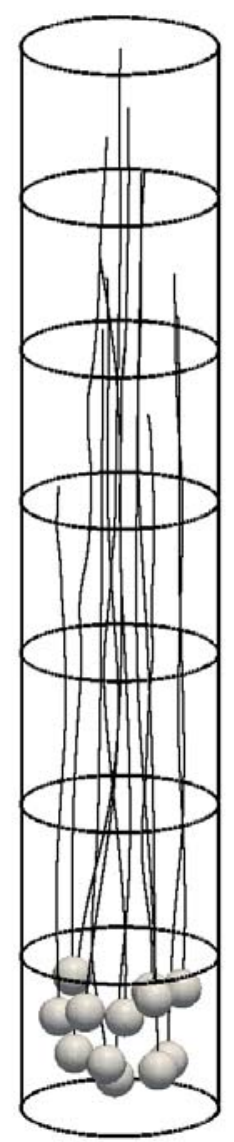

Figure 25: Homogeneous bubbly flow at $E o=3, M=1 \times 10^{-3}, \eta_{\rho}=10$ and $\eta_{\mu}=10$. Bubble trajectories are integrated from Eq. 27 from $t^{*}=[0,36]$.

a stationary free surface, hence, an additional body force was imposed on both fluids to adjust the pressure gradient such that the flow rate of the 


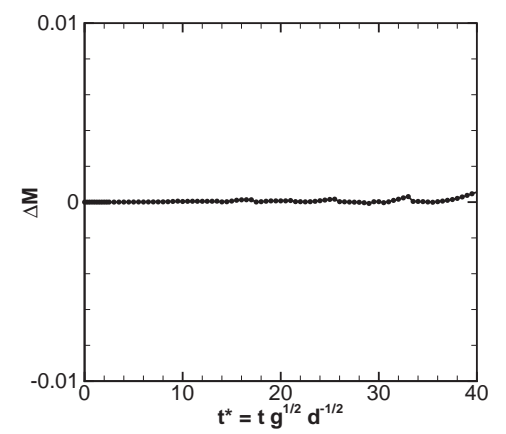

Figure 26: Relative mass error $\Delta M=(M(t)-M(0)) / M(0)$, where $M(t)=\int_{\Omega} \phi_{d}(\mathbf{x}, t) d V$. As reference, $M(40)=6.7 \times 10^{-4}$ after $\sim 200000$ iterations.

fluid phase is exactly zero (Yin and Koch , 2008). Following the work of Bunner and Tryggvason (2002) and Yin and Koch (2008), this force is equivalent to the pressure gradient generated by the base of a flow container which prevents the gravitational force to cause the acceleration of the entire flow field in the downward vertical direction. Indeed, a constant force equal to the space-averaged density times the gravitational acceleration, $\rho_{0} \mathbf{g}$ where $\rho_{0}=\int_{\Omega}\left(\phi_{d}(\mathbf{x}, 0) \rho_{d}+\left(1-\phi_{d}(\mathbf{x}, 0)\right) \rho_{c}\right) d V$, is subtracted from the right hand side of the Navier-Stokes Eq. 1 (see Bunner and Tryggvason (2002)). As a consequence, the velocity field of the bubbles in the whole domain will be oriented upwards. Since both fluids are assumed to be incompressible, the overall volumetric fraction of dispersed fluid phase, $\left\langle\alpha_{d}\right\rangle=2 n_{d} \pi d^{3} /\left(3 \pi D_{\Omega}^{2} H_{\Omega}\right)$, is constant throughout the simulation. A free bubble array of bubbles are initially placed in a quiescent liquid following a random pattern, as shown in Fig. 22a. According to the work of Clift et al. (1978), the gravity-driven bubble motion in an infinite domain can be characterized by the Eötvos number, the Morton number and the ratios of viscosity and density, which are defined in Eq. (17). Physical and geometrical parameters used in this section are summarized in Table 7 . The accuracy of the present level-set method with respect to simulation of single buoyant bubbles has been shown in Balcázar et al. (2014) and it has been found that about 25 grid points per bubble diameter are sufficient for accurate capturing of bubble dynamics. Numerical simulations reported by Balcázar et al. (2014) were performed for flow regimes given by $1 \leqslant E o<100,1 \times 10^{-4} \leqslant M \leqslant 1 \times 10^{3}$ and $R e<30$, 


\begin{tabular}{ll}
\hline Domain size $\left(D_{\Omega}, H_{\Omega}\right)$ & $(5 d, 4 d)$ \\
Mesh: cells per plane & 31518 \\
Mesh: number of planes & 160 \\
Mesh: number of cells & $5.042 \times 10^{6}$ \\
Mesh: cell size $(h)$ & $\approx d / 40$ \\
Density ratio & 10 \\
Viscosity ratio & 10 \\
Number of bubbles & 12 \\
Average void fraction $\left(<\alpha_{d}>\right)$ & 0.032 \\
Eötvos number & 3.0 \\
Morton number & $1 \times 10^{-3}$ \\
\hline
\end{tabular}

Table 7: Physical and geometrical parameters for gravity-driven bubbly flow in a vertical pipe.

which include spherical, ellipsoidal and skirted bubbles. However, in order to improve the resolution of films formed when bubbles collide, 40 grid points per bubble diameter are used in the present work.

The velocity of the bubble centroid is calculated as follows:

$$
\left\langle\mathbf{v}_{i}\right\rangle(t)=\frac{\int_{\Omega} \mathbf{v} \phi_{i}(\mathbf{x}, t) d V}{\int_{\Omega} \phi_{i}(\mathbf{x}, t) d V} \quad \text { for } i=1, . ., n_{d}
$$

whereas the rise velocity of the swarm is computed by:

$$
\left\langle\mathbf{v}_{d}\right\rangle(t)=\frac{1}{n_{d}} \sum_{i=1}^{n_{d}}\left\langle\mathbf{v}_{i}\right\rangle(t)
$$

The bubble rise is characterized by the bubble Reynolds number:

$$
\left\langle R e_{i}\right\rangle=\frac{\rho_{c} d \mathbf{v}_{i} \cdot \mathbf{e}_{y}}{\mu_{c}}
$$

where $\mathbf{e}_{y}$ is a unit vector parallel to $+y$ direction. Finally, the average Reynolds number for the swarm of bubbles is calculated as:

$$
\left\langle R e_{d}\right\rangle=\frac{1}{n_{d}} \sum_{i=1}^{n_{d}}\left\langle R e_{i}\right\rangle
$$

Once the velocity of each bubble has been calculated by Eq. 23, the trajectory of the bubble can be determined by:

$$
\left\langle\mathbf{x}_{i}\right\rangle(t)=\left\langle\mathbf{x}_{i}^{0}\right\rangle+\int_{0}^{t}\left\langle\mathbf{v}_{i}\right\rangle(t) d t
$$


where $\left\langle\mathbf{x}_{i}^{0}\right\rangle$ is the initial position of the $i^{\text {th }}$ bubble centroid.

Examining the velocity of each bubble in Fig. 23a, it is clear that the velocity of the center of mass of the bubble swarm reaches a steady state, while each individual bubble does not, furthermore they present oscillating rise velocities. This transient behavior is produced by the wake interaction of bubbles, moreover, a nonuniform bubble concentration will induce a gradient velocity, which will, in turn generate a lift force on the bubbles that influences the distribution of bubbles. Also, the bubbles near the symmetry axis of the cylinder move faster than the bubbles near the wall. This observation can be confirmed by the bubble trajectories plotted in Fig. 25. It can be seen that the bubbles move, not only in streamwise direction, but also in radial direction, as shown in Fig. 23b. These radial movements tend to align bubbles at approximately constant distance from the wall (see Fig. 23b). A typical interaction event of a bubble-pair is illustrated in Fig. 24, where one bubble accelerates in the wake of a leading bubble until they collide, after which the trailing bubble pushes the leading bubble aside. A similar sequence of images has been reported in Bunner and Tryggvason (2003), to illustrate the so-called "drafting, kissing and tumbling "process. Time evolution of the relative mass error of dispersed phase, is displayed in Fig. 26. The results show small mass error over a considerable integration time. Therefore, the long-time simulation presented in this section demonstrates the feasibility of using the multiple marker level-set approach to examine the dynamics of homogeneous bubbly flows without numerical coalescence.

Currently, the CLS function of each fluid particle is defined as a scalar field in the entire spatial domain $\Omega$, therefore the required memory storage and computational effort which is associated with interface representation scales with $n_{b} n_{h}$ for the multiple marker level-set method, whereas it scales with $n_{h}$ for the conventional level-set method. Here, $n_{b}$ is the number of fluid particles and $n_{h}$ is the number of control volumes. Consequently, the simulation of bubbly flows is practical only for a low number of fluid particles, less than $O(100)$.

\section{Conclusions}

In this paper we have presented a novel numerical method for interface advection which has the additional ability to handle the interaction of multiple deformable fluid particles without numerical coalescence. The multiple marker level-set method, combines a conservative level-set method on un- 
structured grids introduced in Balcázar et al. (2014) with a multiple marker approach, where separated fluid regions of the dispersed phase are represented by different CLS functions. Therefore, multiple interfaces can be solved at the same control volume avoiding their numerical merging.

The results of droplet impact on a liquid-liquid interface confirm that the multiple marker level-set method can be used effectively for the simulation of the interaction of a deformable fluid particle with another interface, accurately representing the surface tension on the dynamics of the flow. Indeed, a close agreement was found between present numerical results and experiments reported by Mohamed-Kassim et al. (2003). Then, numerical predictions of fully three-dimensional collisions of two drops with bouncing outcome were performed. Detailed comparisons with the time-resolved images obtained from the experiments of Pan et al. (2008) were used to validate the numerical method. Both qualitative and quantitative agreements were obtained in terms of droplet shape and phase. Finally, the unstructured multiphase solver has been used to perform numerical simulations of gravitydriven bubbly flow in a vertical pipe with monodisperse and noncoalescing bubbles. To the best of the author's knowledge, this is the first time that an interface multi-tracking approach is used in the context of the conservative level-set method, moreover, numerical investigations of bubble swarms on vertical pipes has not been previously reported. Thus, it is demonstrated that the present numerical method is able to capture the dynamics of multiple deformable fluid particles, avoiding the numerical coalescence inherent to standard formulations of level-set methods. One possible extension of this work would be to vary the number of bubbles and flow conditions to obtain bubble concentration and fluid velocity profiles. These profiles can be used for the determination of closures relations as well as appropriate boundary conditions for the averaged two-fluid equations.

\section{Acknowledgments}

This work has been financially supported by the Ministerio de Economía y Competitividad, Secretaría de Estado de Investigación, Desarrollo e Innovación, Spain (ENE2011-28699), and by Termo Fluids S.L. Néstor Balcázar acknowledges financial support in form of a doctoral scholarship of the Agencia Española de Cooperación Internacional para el Desarrollo (AECID), Spain. 


\section{References}

Balcázar, N., Jofre, L., Lehmkuhl, O.,Castro, J., Rigola, J., 2014. A finitevolume/level-set method for simulating two-phase flows on unstructured grids, International Journal of Multiphase Flow 64, 55-72.

Brackbill, J.U., Kothe, D.B., Zemach, C., 1992. A Continuum Method for Modeling Surface Tension, J. Comput. Phys. 100, 335-354.

Bunner, B., Tryggvason, G., 2002. Dynamics of homogeneous bubbly flows Part 1. Rise velocity and microstructure of the bubbles, J. Fluid Mech. $466,17-52$.

Bunner, B., Tryggvason, G., 2003. Effect of bubble deformation on the properties of bubbly flows, J. Fluid Mech. 495, 77-118.

Chorin, A.J., Numerical solution of the Navier-Stokes equations. 1968. Math. Comput. 22, 745-762.

Ceniceros, H., Roma, A.M., Silveira-Neto, A., Villar, M.M., A Robust, Fully Adaptive Hybrid Level-Set/Front-Tracking Method for Two-Phase Flows with an Accurate Surface Tension Computation. 2010. Commun. Comput. Phys. 8, 51-94.

Clift, R., Grace, J.R., Weber, M.E., Bubbles, Drops and Particles. Academin Press, New York, 1978.

Coyajee, E., Boersma, J.B., Numerical simulation of drop impact on a liquidliquid interface with a multiple marker front-capturing method, 2009, J. Comp. Phys. 228, 4444-4467

Deen, N.G., Van Sint Annaland, M., Kuipers, J.A.M. , 2009. Direct numerical simulation of complex multi-fluid flows using a combined front tracking and immersed boundary method, Chemical Engineering Science 64, 2186-2201.

Dijkhuizen, W., Roghair, I., Van Sint Annaland, M., Kuipers, J.A.M., 2008. DNS of gas bubbles behaviour using an improved 3D front tracking model Drag force on isolated bubbles and comparison with experiments, Chemical Engineering Science 65, 1415-1426

Esmaeeli, A., Tryggvason, G., 2005. A DNS study of the buoyant rise of bubbles at O(100) Reynolds number, Physics of Fluids 17, 093303. 
Felten, F.N., Lund, T.S., 2006. Kinetic energy conservation issues associated with the collocated mesh scheme for incompressible flow, J. Comput. Phys. $215,465-484$

Focke, C., Kuschel, M., Sommerfeld, M., Bothe, D., 2006. Collision between high and low viscosity droplets: Direct Numerical Simulations and experiments, International Journal of Multiphase Flow 56, 81-92

Gottlieb, S., Chi-Wang, S., 1998. Total Variation Dimishing Runge-Kutta Schemes, Mathematics of Computations 67, 73-85.

Guermond, J.L., Minev, P., Jie, S., 2006. An overview of projection methods for incompressible flows, Comput. Methods Appl. Mech. Engrg. 195, 60116045 .

Harten, A., 1977. The Artificial Compression Method for Computation of Shocks and Dontact Discontinuities: I. Single conservation laws, Comm. Pure Appl. Math., 611-638.

Jamet, D., Lebaigue, O., Morel, C., Arcen, B., 2010. Towards a multi-scale approach of two-phase flow modeling in the context of DNB modeling, Nuclear Engineering and Design 240, 2131-2138.

Jiang, X., James, A., 2007. Numerical simulation of the head-on collision of two equal sized drops with van der Waals forces, Journal of Engineering Mathematics 59, 99-121.

Kim, B., 2010. Multi-Phase Fluid Simulations Using Regional Level Sets, SIGGRAPH Asia, , ACM Transactions on Graphics (TOG), Volume 29, Issue 5 .

Kwakkel, M., Breugem, W.P., Boersma, B., 2013. Extension of a CLSVOF method for droplet-laden flows with a coalescence/breakup model, Journal of Computational Physics 253, 166188.

Lehmkuhl, O., Perez-Segarra, C.D., Soria, M., Oliva, A., 2007, A new Parallel unstructured CFD code for the simulation of turbulent industrial problems on low cost PC cluster, Proceedings of the Parallel CFD 2007 Conference, pp.1-8.

Lamb, H., 1932. Hydrodynamics, Dover, New York, 1932. 
Li, X., Fritsching, U., 2011. Numerical investigation of binary droplet collisions in all relevant collision regimes, The Journal of Computational Multiphase Flows 3, 207224.

Losasso, F., Shinar, T. Selle, A., Fedkiw, R., 2006. Multiple Interacting Liquids, SIGGRAPH, ACM TOG 25, 812-819.

Lu, J., Fernández, A., Tryggvason, G., 2005. The effect of bubbles on the wall drag in a turbulent channel flow, Physics of Fluids 17, 095102.

Magnaudet, J., Eames, I., 2000. The Motion of High-Reynolds-Number Bubbles in Inhomogeneous Flows, Annu. Rev. Fluid Mech. 32, 659-708.

Merriman, B., Bence, J., Osher, S., 1994. Motion of multiple junctions: A level set approach, J. Comput. Phys. 112, 334-363.

Mohamed-Kassim, Z., Longmire, E.K., Drop impact on a liquid-liquid interface, 2003. Phys. Fluids 15, 3263-3273.

Mohamed-Kassim, Z., Longmire, E.K., Kim, J.S., Zheng, X., Lowengrub, J., Experiments and Computations on Drop Impact at a Liquid/Liquid Interface, 2004. 5th International Conference on Multiphase Flow, Paper 122.

Mudde, R., 2005. Gravity-Driven bubbly flows, Annu. Rev. Fluid Mech. 37, 393-423.

Nobari, M.R., Tryggvason, G., 1996. Numerical Simulations of ThreeDimensional Drop Collisions, AIAA Journal 34, 750-755.

Nobari, M. R. and Jan, Y.J. and Tryggvason, G., 1996. Head-on collision of drops-A numerical investigation, Phys. Fluids 8, 29-42.

Olsson, E., Kreiss, G., 2005. A conservative level set method for two phase flow, J. Comput. Phys. 210, 225-246.

Olsson, E., Kreiss, G., 2007. A conservative level set method for two phase flow II, J. Comput. Phys. 225, 785-807.

Osher, S., Sethian, J.A., 1988. Fronts propagating with curvature-dependent speed: Algorithms based on Hamilton-Jacobi formulations, J. Comput. Phys. 79, 175-210. 
Pan, K., Law, C.K., Zhou, B., 2008. Experimental and mechanistic description of merging and bouncing in head-on binary droplet collision, Journal of Applied Physics 103, 064901.

Qian, J., Law, C.K., 1997. Regimes of coalescence and separation in droplet collision, Journal of Fluid Mechanics 331, 59-80.

Ruuth, S., 1998. A diffusion-generated approach to multiphase motion, J. Comput. Phys. 145, 166-192.

Rhie, C.M., Chow, W.L., 1983. Numerical Study of the Turbulent Flow past an Airfoil with Trailing Edge Separation, AIAA J. 21, 1525-1532.

Rodríguez, I., Borrell, R., Lehmkuhl, O., Pérez-Segarra, C.D., Oliva, A., 2011. Direct numerical simulation of the flow over a sphere at $R e=3700$, J. Fluids Mech. 679, 263287.

Roghair, I., Lau, Y.M., Deen, N.G., Slagter, H.M., Baltussen, M.W., Van Sint Annaland, M., Kuipers, J.A.M., 2011. On the drag force of bubbles in bubble swarms at intermediate and high Reynolds numbers. Chemical Engineering Science 66, 3204-3211.

Sanada, T., Sato, A., Shirota, M., Watanabe, M., 2009. Motion and coalescence of a pair of bubbles rising side by side, Chem. Eng. Sci. 64, 2659-2671.

Sankaranarayanan, K., Shan, X., Kevrekidis, I.G., Sundaresan, S., 2002. Analysis of drag and virtual mass forces in bubbly suspensions using an implicit formulation of the lattice Boltzmann method, J. Fluid Mech. 452, 61-96.

Scardovelli, R., Zaleski, S., 1999. Direct numerical simulation of free surface and interfacial flow, Annu. Rev. Fluid Mech. 31, 567-603.

Smith, K., Solis, F., Chopp, D., 2002. A projection method for motion of triple junctions by level sets, Interfaces and Free Boundaries 3, 263-276.

Sussman, M., Puckett, E., 2000. A Coupled Level Set and Volume-of-Fluid Method for Computing 3D and Axisymmetric Incompressible Two-Phase Flows, J. Comput. Phys. 162, 301-337. 
Sussman, M., Smereka, P., Osher, S., 1994. A Level Set Approach for Computing Solutions to Incompressible Two-Phase Flow, J. Comput. Phys. 144, 146-159.

Sweby, P.K., 1984. High Resolution Using Flux Limiters for Hyperbolic Conservation Laws, SIAM Journal on Numerical Analysis 21, 995-1011.

Tran, T., Maleprade, H., Sun, C., Lohse, D., 2013. Air entrainment during impact of droplets on liquid surfaces, J. Fluid Mech. 726, R3.

Thomas, S., Esmaeeli, A., Tryggvason, G., 2010. Multiscale computations of thin films in multiphase flows, International Journal of Multiphase Flow 36,7177 .

Trias, F.X., Lehmkuhl, O., 2011. A Self-Adaptive Strategy for the Time Integration of Navier-Stokes Equations. Numerical Heat Transfer, Part B $60,116-134$

Tryggvason, G., Dabiri, S., Abouhasanzadeh, B., Jaicai, L., 2013. Multiscale considerations in direct numerical simulations of multiphase flows, Phys. Fluids 25, 031302.

Tryggvason, G., Bunner, B., Esmaeeli, A., Juric, D., Al-Rawahi, N., Tauber, W., Han, J., Nas, S., Jan, Y-J., 2001. A Front-Tracking Method for the Computations of Multiphase Flow, J. Comput. Phys. 169, 708-759.

Tryggvason, G., Thomas, S., Lu, J., Aboulhasanzadeh, B., 2001. A FrontTracking Method for the Computations of Multiphase Flow, J. Comput. Phys. 169, 708-759.

Unverdi, S., Tryggvason, G., 1992. A front-tracking method for viscous, incompressible, multifluid flows, J. Comput. Phys. 100, 25-37.

Van Sint Annaland, M., Deen, N.G., Kuipers, J.A.M., 2005. Numerical Simulation of gas bubbles behaviour using a three-dimensional volume-of-fluid method, Chemical Engineering Science 60, 2999-3011.

Wang, M.Y., Wang, X., 2004. "Color" level sets: a multi-phase method for structural topology optimization with multiple materials, Computer Methods in Applied Mechanics and Engineering Volume 193, Issues 6-8, 469-496. 
Yin, X. and Koch, L., 2008. Lattice-Boltzmann simulation of finite Reynolds number buoyancy-driven bubbly flows in periodic and wall domains, Physics of fluids 20, 103304.

Zheng, X., Lowengrub, J., Anderson, A., Cristini, V., 2005. Adaptive unstructured volume remeshing II: Application to two and three dimensional level-set simulations of multiphase flow, Journal of Computational Physics 208, 626-650. 